DOI: http://dx.doi.org/10.18273/revint.v37n1-2019006

\title{
On the existence of a priori bounds for positive solutions of elliptic problems, II
}

\author{
ROSA PARDO* \\ Universidad Complutense de Madrid, Departamento de Análisis Matemático y \\ Matemática Aplicada, Madrid, Spain.
}

Abstract. We continue studying the existence of uniform $L^{\infty}$ a priori bounds for positive solutions of subcritical elliptic equations

$$
(\mathcal{P})_{p} \quad-\Delta_{p} u=f(u), \text { in } \Omega, \quad u=0, \text { on } \partial \Omega,
$$

We provide sufficient conditions for having a-priori $L^{\infty}$ bounds for $C^{1, \mu}(\bar{\Omega})$ positive solutions to a class of subcritical elliptic problems in bounded, convex, $C^{2}$ domains. In this part II, we extend our results to Hamiltonian elliptic systems $-\Delta u=f(v),-\Delta v=g(u)$, in $\Omega, u=v=0$ on $\partial \Omega$, when $f(v)=v^{p} /[\ln (e+v)]^{\alpha}, g(u)=u^{q} /[\ln (e+u)]^{\beta}$, with $\alpha, \beta>2 /(N-2)$, and $p, q$ are lying in the critical Sobolev hyperbolae $\frac{1}{p+1}+\frac{1}{q+1}=\frac{N-2}{N}$. For quasilinear elliptic equations involving the $p$-Laplacian, there exists a-priori bounds for positive solutions of $(\mathcal{P})_{p}$ when $f(u)=u^{p^{\star}-1} /[\ln (e+u)]^{\alpha}$, with $p^{*}=N p /(N-p)$, and $\alpha>p /(N-p)$. We also study the asymptotic behavior of radially symmetric solutions $u_{\alpha}=u_{\alpha}(r)$ of $(\mathcal{P})_{2}$ as $\alpha \rightarrow 0$.

Keywords: A priori estimates, subcritical nonlinearity, moving planes method, Pohozaev identity, critical Sobolev hyperbola, biparameter bifurcation.

MSC2010: 35B45, 35J92, 35B33, 35J47, 35J60, 35J61.

\section{Sobre la existencia de cotas a priori para soluciones positivas de problemas elípticos, II}

Resumen. Continuamos estudiando la existencia de cotas uniformes a priori para soluciones positivas de equaciones elípticas subcríticas

$$
(\mathcal{P})_{p} \quad-\Delta_{p} u=f(u), \text { en } \Omega, \quad u=0, \text { sobre } \partial \Omega,
$$

Proporcionamos condiciones suficientes para que las soluciones positivas en $C^{1, \mu}(\bar{\Omega})$ de una clase de problemas elípticos subcríticos tengan cotas a-priori $L^{\infty}$ en dominios acotados, convexos, y de clase $C^{2}$.

\footnotetext{
*E-mail: rpardo@mat.ucm.es

Received: 15 November 2018, Accepted: 20 December 2018.

To cite this article: R. Pardo, On the existence of a priori bounds for positive solutions of elliptic problems,

I, Rev. Integr. temas mat. 37 (2019), No. 1, 113-148. doi: 10.18273/revint.v37n1-2019006.
} 
En esta parte II, extendemos nuestros resultados a sistemas elípticos Hamiltonianos $-\Delta u=f(v),-\Delta v=g(u)$, en $\Omega, u=v=0$ sobre $\partial \Omega$, cuando $f(v)=v^{p} /[\ln (e+v)]^{\alpha}, g(u)=u^{q} /[\ln (e+u)]^{\beta}$, con $\alpha, \beta>2 /(N-2)$, у $p, q$ varían sobre la hipérbola crítica de Sobolev $\frac{1}{p+1}+\frac{1}{q+1}=\frac{N-2}{N}$. Para ecuaciones elípticas cuasilineales que involucran al operador $p$-Laplacian, existen cotas a-priori para soluciones positivas de $(\mathcal{P})_{p}$ en el espacio $C^{1, \mu}(\bar{\Omega}), \mu \in(0,1)$, cuando $f(u)=u^{p^{\star}-1} /[\ln (e+u)]^{\alpha}$, con $p^{*}=N p /(N-p)$, y $\alpha>p /(N-p)$.

También estudiamos el comportamiento asintótico de soluciones radialmente simétric $u_{\alpha}=u_{\alpha}(r)$ de $(\mathcal{P})_{2}$ cuando $\alpha \rightarrow 0$.

Palabras clave: Estimaciones a priori, no-linealidades subcríticas, método de "moving planes", igualdad de Pohozaev, hipérbola crítica de Sobolev, bifurcación biparamétrica.

\section{Introduction}

In this part II, we describe our a priori bounds results on semilinear elliptic systems, on quasilinear elliptic equations for the $p$-laplacian, and the asymptotic behavior of radial solutions as $\alpha \rightarrow 0^{+}$in several subsections. We leave the proofs for the following sections.

In a previous paper containing part I, when $p=2$, we show the existence of $L^{\infty} a$-priori bounds for classical, positive solutions of semilinear elliptic equations $-\Delta u=f(u)$ with Dirichlet homogeneous boundary conditions, for

$$
f(u)=\frac{u^{2^{*}-1}}{[\ln (e+u)]^{\alpha}}, \quad \text { with } 2^{*}=\frac{2 N}{N-2}, \quad \text { and } \alpha>\frac{2}{N-2} .
$$

Appealing to the Kelvin transform, we extend our results to non-convex domains (see [16], [17]).

\subsection{Hamiltonian elliptic systems}

We provide a-priori $L^{\infty}(\Omega)$-bounds for classical positive solutions of Hamiltonian elliptic systems

$$
\left\{\begin{aligned}
-\Delta u & =f(v), & & \text { in } \Omega, \\
-\Delta v & =g(u), & & \text { in } \Omega, \\
u=v & =0, & & \text { on } \partial \Omega,
\end{aligned}\right.
$$

where $\Omega \subset \mathbb{R}^{N}, N \geq 2$, is a bounded, convex $C^{3}$ domain,

$$
f(v)=\frac{v^{p}}{[\ln (e+v)]^{\alpha}}, \quad g(u)=\frac{u^{q}}{[\ln (e+u)]^{\beta}}, \quad \alpha, \beta>\frac{2}{N-2},
$$

and $p, q$, are in the so called critical Sobolev hyperbola

$$
\frac{1}{p+1}+\frac{1}{q+1}=\frac{N-2}{N}
$$

(see [71] and Theorem 1.1).

[Revista Integración, temas de matemáticas 
Using these a priori bounds, and local and global bifurcation techniques, we prove the existence of positive solutions for a corresponding parametrized semilinear elliptic system (see [71] and Theorem 1.3).

If the exponents $\alpha=\beta=0$, then we have the Lane-Emden system

$$
\left\{\begin{array}{l}
-\Delta u=v^{p}, \quad \text { in } \Omega, \\
-\Delta v=u^{q}, \quad \text { in } \Omega, \\
u=0, \quad v=0 \quad \text { on } \partial \Omega
\end{array}\right.
$$

where $\Omega \subset \mathbb{R}^{N}$ is either a bounded, smooth subset, or a half space, or $\mathbb{R}^{N}$. The exponents $(p, q)$ play a crucial role for the existence or nonexistence of positive solutions of LaneEmdem system, depending on the boundedness or not of $\Omega$. When $\Omega$ is a bounded smooth star-shaped domain, the Sobolev hyperbola divides, on the $p q$-plane, existence and nonexistence of positive solutions of the Lane-Emdem system (see fig. 1; we include the details in Section 2). When $\Omega=\mathbb{R}^{N}$ there is a conjecture: the hyperbola (2) is also the dividing curve between existence and nonexistence for the Lane-Emdem system.

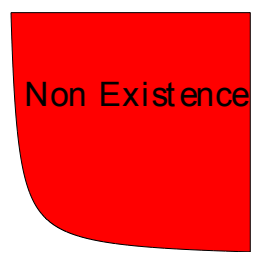

Figure 1. $\Omega$ bounded

We now state our main results on elliptic systems. See Section 2, or [71, Theorems 1.1, $1.3]$ for a proof.

Theorem 1.1 (a-priori $L^{\infty}$ bounds). Let us consider the following semilinear elliptic system:

$$
\left\{\begin{array}{l}
-\Delta u=\frac{v^{p}}{[\ln (e+v)]^{\alpha}}, \quad \text { in } \Omega, \\
-\Delta v=\frac{u^{q}}{[\ln (e+u)]^{\beta}}, \quad \text { in } \Omega, \\
u=0, \quad v=0 \quad \text { on } \partial \Omega,
\end{array}\right.
$$

where $\Omega \subset \mathbb{R}^{N}, N \geq 3$, is a bounded, convex domain with boundary $\partial \Omega$ of class $C^{2}$. Suppose that $p, q>1, \alpha, \beta>\frac{2}{N-2}, p, q$ are on the critical Sobolev hyperbola (2), and assume that

$$
\min \left\{\frac{p}{\alpha}, \frac{q}{\beta}\right\} \geq \max _{t \geq 0}\left[\frac{t}{(e+t) \ln (e+t)}\right] \text {. }
$$

Vol. 37, $\left.\mathrm{N}^{\circ} 1,2019\right]$ 
Then there exists a uniform constant $C$, depending only on $\Omega$ and $p, q, \alpha, \beta$ but not on $(u, v)$, such that

$$
\|u\|_{L^{\infty}(\Omega)} \leq C \text { and }\|v\|_{L^{\infty}(\Omega)} \leq C,
$$

for all positive solutions $(u, v)$ of $(4)$.

Remark 1.2. Observe that condition (2) relates the exponents $p$ and $q$ to the Sobolev hyperbola, and condition (5) ensures that our nonlinearities are nondecreasing since it will be needed in the proof. Notice that

$$
\max _{t \geq 0}\left[\frac{t}{(e+t) \ln (e+t)}\right]=\frac{e}{e+t^{*}},
$$

where $t^{*}$ is the solution of the logarithmic equation $e \ln (e+t)=t$.

In the next theorem, we state the existence of positive solutions for the semilinear biparameter elliptic systems (4).

Theorem 1.3 (Existence). Consider the biparameter elliptic system

$$
\left\{\begin{array}{l}
-\Delta u=\lambda v+\frac{v^{p}}{[\ln (e+v)]^{\alpha}}, \quad \text { in } \Omega, \\
-\Delta v=\mu u+\frac{u^{q}}{[\ln (e+u)]^{\beta}}, \quad \text { in } \Omega, \\
u=0, \quad v=0 \quad \text { on } \partial \Omega,
\end{array}\right.
$$

where the exponents $p, q, \alpha, \beta$ are as defined in Theorem 1.1, and the parameters $\lambda$ and $\mu$ are non-negative real parameters.

Then (6) has a positive solution $(u, v)$ if, and only if, $\lambda \mu<\lambda_{1}^{2}$, where $\lambda_{1}$ is the principal eigenvalue associated with the linear eigenvalue problem with homogeneous Dirichlet boundary conditions $-\Delta \phi=\lambda \phi \quad$ in $\Omega ; \phi=0$ on $\partial \Omega$.

Proof. See Section 2, or [71, Theorem 1.3] for a proof.

The ideas of the proof of Theorem 1.1 are adapted for systems. Moving planes for system provides $L^{\infty}$ bounds in a neighborhood of the boundary of a convex domain, for classical positive solutions of (1). Rellich-Pohazev identity for systems give us two bounded integrals in $\Omega$. With the help of Morrey's Theorem, we estimate the radius $R,\left(R^{\prime}\right)$, of a ball where the function $u,(v)$, exceeds half of its $L^{\infty}$ bound. Then, we get a lower bound of the above integrals, deriving the $L^{\infty}$ bounds for classical positive solutions of (4).

\subsection{The $p$-Laplacian}

We consider the Dirichlet problem for positive solutions of the equation $-\Delta_{p}(u)=f(u)$ in a convex, bounded, smooth domain $\Omega \subset \mathbb{R}^{N}$, with $f$ locally Lipschitz continuous. We

provide sufficient conditions guarantying $L^{\infty}$ a priori bounds for positive solutions of

[Revista Integración, temas de matemáticas 
some elliptic equations involving the $p$-Laplacian and extend the class of known nonlinearities for which the solutions are $L^{\infty}$ a priori bounded. As a consequence we prove the existence of positive solutions in convex bounded domains.

Specifically, in case of elliptic equations involving the $p$-Laplacian, we prove the existence of a-priori bounds for $C^{1, \mu}(\bar{\Omega})$ positive solutions of elliptic equations

$$
-\Delta_{p} u=f(u) \quad \text { in } \Omega, \quad u=0, \quad \text { on } \partial \Omega,
$$

when

$$
f(u)=\frac{u^{p^{\star}-1}}{[\ln (e+u)]^{\alpha}}, \quad \text { with } p^{*}=\frac{N p}{N-p}, \quad \text { and } \alpha>\frac{p}{(N-p)},
$$

see Example 1 in [31].

Corollary 1.4. Assume that $\Omega \subset \mathbb{R}^{N}$ is a smooth bounded convex domain, $1<p<N$, and $u>0$ is a $C^{1}(\bar{\Omega})$ solution to

$$
\left\{\begin{aligned}
-\Delta_{p} u & =\frac{u^{p^{\star}-1}}{[\ln (e+u)]^{\alpha}}, & & \text { in } \Omega, \\
u & =0, & & \text { on } \partial \Omega,
\end{aligned}\right.
$$

with $\alpha>p /(N-p)$.

Then, there exists a uniform constant $C$, depending only on $\Omega$ and $f$ but not on the solution, such that $\|u\|_{L^{\infty}(\Omega)} \leq C$.

Proof. It is a Corollary of Theorem 3.5 (see also [31, Theorem 1.7 and Example 1 in p. 491]).

\subsection{Final remarks}

We finally provide sufficient conditions for a uniform $L^{2^{\star}}(\Omega)$ bound to imply a uniform $L^{\infty}(\Omega)$ bound, for positive classical solutions to a class of subcritical elliptic problems in bounded $C^{2}$ domains. We also establish an equivalent result for sequences of boundary value problems.

We also study the asymptotic behavior of radially symmetric solutions $u_{\alpha}=u_{\alpha}(r)$ to the subcritical semilinear elliptic problem

$$
(P)_{\alpha}\left\{\begin{aligned}
-\Delta u & =u^{\frac{N+2}{N-2}} /[\log (e+u)]^{\alpha} & & \text { in } \Omega=B_{R}(0) \subset \mathbb{R}^{N}, \\
u & >0, & & \text { in } \Omega, \\
u & =0, & & \text { on } \partial \Omega,
\end{aligned}\right.
$$

as $\alpha \rightarrow 0^{+}$. We prove that there exists an explicitly defined constant $L(N, R)>0$, only depending on $N$ and $R$, such that

$$
\begin{aligned}
\limsup _{\alpha \rightarrow 0^{+}} \frac{\alpha u_{\alpha}(0)^{2}}{\left[\log \left(e+u_{\alpha}(0)\right)\right]^{1+\frac{\alpha(N+2)}{2}}} & \leq L(N, R) \\
& \leq 2^{*} \liminf _{\alpha \rightarrow 0^{+}} \frac{\alpha u_{\alpha}(0)^{2}}{\left[\log \left(e+u_{\alpha}(0)\right)\right]^{\frac{\alpha(N-4)}{2}}},
\end{aligned}
$$

Vol. 37, $\mathrm{N}^{\circ}$ 1, 2019] 


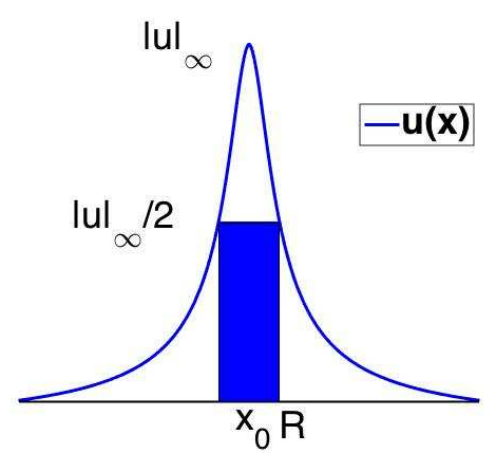

Figure 2. Let $(u, v)$ be a solution of (1); we plot one component $u$, its $L^{\infty}$ norm, and the estimate of the radius $R$ such that $u(x) \geq \frac{\|u\|_{\infty}}{2}$ for all $x \in B\left(x_{0}, R\right)$, where $x_{0}$ is such that $u\left(x_{0}\right)=\|u\|_{\infty}$.

see $[75]$.

This paper is organized in the following way. Section 2 is devoted to semilinear elliptic systems. In Section 3 we state an abstract theorem on a priori bounds for $p$-laplacian equations in convex domains. Section 4 contains a result on the equivalence between uniform $L^{2^{\star}}(\Omega)$ a-priori bounds and uniform $L^{\infty}(\Omega)$ a-priori bounds for subcritical elliptic equations. In Section 5 we study the asymptotic behavior of radially symmetric solutions $u_{\alpha}=u_{\alpha}(r)$ as $\alpha \rightarrow 0^{+}$. In Section 6 we collect some open problems.

\section{A priori bounds and existence of positive solutions for semilinear elliptic systems}

It is natural to ask whether it is possible to obtain the corresponding a priori results for systems. In this Section, we extend the results of [16] from scalar equations to systems. The existence of a-priori bounds for the system (4) is proved in the same lines of [16], that is, using the Rellich-Pohozaev identity and the method of moving planes as in [21], combined with Morrey's Theorem. The moving planes method is used to obtain $L^{\infty}$ bounds in a neighborhood of the boundary for classical positive solutions of (4), whereas the Rellich-Pohozaev identity is used to get two bounded integrals in $\Omega$. Furthermore, Morrey's Theorem is used to estimate the radius $R,\left(R^{\prime}\right)$, of a ball where the function $u,(v)$, exceeds half of its $L^{\infty}$ bound (see fig. 2), allowing us to reach a contradiction on the lower bounds of the above integrals.

We consider the semilinear elliptic system given by (4) where $\Omega \subset \mathbb{R}^{N}, N \geq 3$, is a bounded, convex domain with a smooth boundary $\partial \Omega$ (at least of class $C^{3}$ ), and $1<p, q<\infty, \alpha, \beta>0$. The purpose of this Section is to establish a-priori estimates for positive classical solutions of (4) and subsequently prove an existence result for the parametrized version for the system. By a positive classical solution of (4), we mean $(u, v)$ that satisfies $(4)$ and both components are positive. Let us mention that when the 
exponents $\alpha=\beta=0$, we have the system

$$
\left\{\begin{array}{l}
-\Delta u=v^{p}, \quad \text { in } \Omega, \\
-\Delta v=u^{q}, \quad \text { in } \Omega, \\
u=0, \quad v=0 \quad \text { on } \partial \Omega,
\end{array}\right.
$$

that is usually referred to as the Lane-Emden system. This problem arises in modeling spatial phenomena in a variety of biological and chemical problems. Naturally positive solutions of system (7) is of particular interest, and there have been significant studies of positive solutions of ( 7 ) where $\Omega$ is either a bounded, smooth subset of $\mathbb{R}^{N}$, a half space, or the entire space $\mathbb{R}^{N}$ (see [11], [12], [14], [21], [23], [40], [39], [72], [76], [79], [86], [87], [92] and references therein).

It is known that the pair of exponents $(p, q)$ plays a crucial role in the questions of existence and nonexistence of positive solutions of (7). For instance, it has been shown that on a bounded smooth star-shaped domain $\Omega \subset \mathbb{R}^{N}$, the Sobolev hyperbola (2) is precisely the dividing curve on the $p q$-plane between existence and nonexistence of positive solutions of (7) (see [21], [72], [76]).

In [21], the authors established a-priori estimates and proved the existence of positive solutions of $(7)$ when $(p, q)$ is subcritical (i.e. $(p, q)$ lies below the critical Sobolev hyperbola), that is, $\frac{1}{p+1}+\frac{1}{q+1}>\frac{N-2}{N}$. Moreover, in [72], the author proved that if $(p, q)$ is critical (i.e. $(p, q)$ lies on critical Sobolev hyperbola) or supercritical (i.e. $(p, q)$ lies above the critical Sobolev hyperbola), namely if $\frac{1}{p+1}+\frac{1}{q+1} \leq \frac{N-2}{N}$, then (7) has no positive solution (see fig. 1).

When $\Omega=\mathbb{R}^{N}$, it has been conjectured that the hyperbola (2) is also the dividing curve between existence and nonexistence for (7). The conjecture has been completely proved for radial positive solutions (see e.g. [72], [85]), that is, if $(p, q)$ is subcritical, then there are no radial positive classical solution to (7) (see [72] for $p>1, q>1$ ) and it has been extended in [85] for the case $p>0$ and $q>0$. Furthermore, if $(p, q)$ is critical or supercritical, system (7) does admit (bounded) positive radial solutions (see e.g. [72], [85]). In the more general case, i.e. without assuming radial symmetry, the question has not been completely answered yet. Partial answers are known for nonexistence of positive entire solutions of (7) when the pair of exponents are subcritical; for example, it has been proved the nonexistence in certain space dimensions [72], [87] or in certain subregions, below the critical hyperbola in the $(p, q)$-plane (see e.g. [14], [87]). For $\Omega=\mathbb{R}_{+}^{N}$ (i.e. the half space), we refer to [11] for the study of nonexistence of positive solutions. These nonexistence results in $\mathbb{R}^{N}$ or $\mathbb{R}_{+}^{N}$ allow to prove a-priori bounds for positive solutions of semilinear elliptic equations in bounded domains via the blow-up method (see e.g. [52], [53], [11], [92]).

In the present Section, we use the method of moving planes and the Rellich-Pohazev identity for systems to establish a-priori $L^{\infty}$ bounds when the pair of exponents $(p, q)$ lies on the critical Sobolev hyperbola (2) and $\alpha, \beta>\frac{2}{N-2}$, and then subsequently prove an existence result for the parametrized version for the system (see system (6) below). Problems of type (4) has been considered by several authors (we refer to [37], [22]). In

Vol. 37, $\left.\mathrm{N}^{\circ} 1,2019\right]$ 
[37, Theorem 1.3] the authors study the existence of solutions of (4) when the pair of exponents $(p, q)$ lies on the critical Sobolev hyperbola (2) and $\alpha<0, \beta>\frac{q+1}{p+1}|\alpha|$, that is, one nonlinearity is above the power function. Whereas in [22, Theorem 2.7] the authors study related nonlinearities when the pair of exponents $(p, q)$ lies below the critical Sobolev hyperbola (2), using variational approaches.

Throughout this Section we assume that $\Omega \subset \mathbb{R}^{N}$ is a smooth bounded, convex domain. The hypothesis on convexity of the domain is needed in order to establish a priori bounds in a neighborhood of the boundary, via the moving planes method (see Lemma 2.1). In [89, Lemma 4.3] the author develop the moving planes method for systems assuming that both nonlinearities are nondecreasing and do not depend explicitly on the spatial variable $x$.

Let us first briefly recall the different methods for deriving a-priori estimates for elliptic systems in the literature. In [93], the existence of a-priori estimates of classical positive solutions of (4) was proved based on scaling or blow-up method which requires the nonlinearities to have a precise asymptotic behavior at infinity, typically $f \sim v^{p}, g \sim u^{q}$. In [79], [80], the authors derived a-priori estimates results using a method close to the Hardy-Sobolev inequalities method. This method requires only upper bounds on the growth of the nonlinearities and allows the nonlinearities to depend on $x, u$ and $v$, but the growth bounds assumed on $f, g$ are more restrictive than in general.

For elliptic equations on general bounded domains, not necessarily convex, de Figueiredo, Lions and Nussbaum [40] applied the moving planes method on the Kelvin transform in order to avoid the difficulty of an empty cap (see [51], [16] for details and the definition of a cap). In that situation, it turns out that the (transformed) nonlinearity depends on the spatial variable $x$. Then, they obtained a priori bounds in a neighborhood of the boundary for classical positive solutions of scalar equations on non-convex domains. To the best of our knowledge, the moving planes method for systems is not yet developed for nonlinearities depending also on the variable $x$. Hence, we focus on convex domains.

The ideas of the proof of Theorem 1.3 are based on local bifurcation techniques [24], combined with global bifurcation theorem [34], [63], [82], and the a-priori estimates. From the seminal works of Crandall and Rabinowitz (see [24], [82]), there are a huge amount of references corresponding to one-parameter bifurcation theory. There are not so many on multiparameter bifurcation. Let us mention Alexander and Antman's Theorem [3] on global multiparameter bifurcation techniques, looking for a change of fixed point index, and providing a manifold of solutions of topological dimension at least the number of parameters, [64] on local multiparameter bifurcation techniques on elliptic systems, [48], [63], [64], [65], [66], [67], [68] on combination of local and global multiparameter bifurcation techniques on elliptic systems, and [49] on the multiparameter bifurcation for the $p$-laplacian.

In this Section, we state two lemmas that are relevant in order to obtain the a priori estimates. The first lemma provides $L^{\infty}$ a priori bounds for any positive solution of (4) in a neighborhood of the boundary (see [40]). The hypothesis of convexity of the domain is needed in order to establish these a priori bounds in a neighborhood of the boundary. Whereas the second lemma provides a Rellich-Pohozaev-Mitidieri type identity (see [72] for a proof). 
Lemma 2.1. Let $(u, v)$ be a positive classical solution of the system (4). Assume that the hypotheses of Theorem 1.1 are satisfied. Then there exists a constant $\delta>0$ depending only on $\Omega$ and not on $p, q, \alpha, \beta$ or $(u, v)$, and a constant $C$ depending only on $\Omega$ and $p, q, \alpha, \beta$, but not on $(u, v)$, such that

$$
\max _{\Omega \backslash \Omega_{\delta}} u \leq C \quad \text { and } \quad \max _{\Omega \backslash \Omega_{\delta}} v \leq C,
$$

where $\Omega_{\delta}:=\{x \in \Omega: d(x, \partial \Omega)>\delta\}$.

The proof is done in a similar way as step 2 in the proof of Theorem 1.1 in [40], using the moving planes method for systems [89, Lemma 4.3]. See also step 1 and 2 in the proof of Theorem 2.1 in [21].

Lemma 2.2 (Rellich-Pohozaev-Mitidieri type identity). Let $u$ and $v$ be in $C^{2}(\bar{\Omega})$, where $\Omega$ is a $C^{1}$ domain in $\mathbb{R}^{N}$, and $u=v=0$ on $\partial \Omega$. Then,

$$
\begin{aligned}
\int_{\Omega} \Delta u(x \cdot \nabla v)+\Delta v(x \cdot \nabla u) & =(N-2) \int_{\Omega}(\nabla u \cdot \nabla v)+\int_{\partial \Omega} \frac{\partial u}{\partial n}(x \cdot \nabla v) \\
& +\int_{\partial \Omega} \frac{\partial v}{\partial n}(x \cdot \nabla u)-\int_{\partial \Omega}(\nabla u, \nabla v)(x \cdot n),
\end{aligned}
$$

where $n$ denotes the exterior normal, and $(x \cdot n)$ denotes the inner product.

The proof of Theorem 1.1 and Theorem 1.3 can be read in [71]; we include it below by completeness.

Proof of Theorem 1.3. Step 1. It follows from (8) and de Giorgi-Nash type Theorems for systems (see [59, Theorem 3.1, p. 397]) that

$$
\|(u, v)\|_{C^{0, \alpha}\left(\Omega_{\delta / 8} \backslash \Omega_{7 \delta / 8}\right)} \leq C, \text { for any } \alpha \in(0,1),
$$

where $\Omega_{t}:=\{x \in \Omega: d(x, \partial \Omega)>t\}$, and $\|(u, v)\|:=\|u\|+\|v\|$. Using Schauder interior estimates (see [54, Theorem 6.2]),

$$
\|(u, v)\|_{C^{2, \alpha}\left(\Omega_{\delta / 4} \backslash \Omega_{3 \delta / 4}\right)} \leq C .
$$

Finally, combining $L^{p}$ estimates with Schauder boundary estimates (see [13], [54], [59]),

$$
\|(u, v)\|_{W^{2, p}\left(\Omega \backslash \Omega_{\delta / 2}\right)} \leq C, \quad \text { for any } \quad p \in(1, \infty) .
$$

By the Sobolev embedding for $p>N$, we have that there exists two constants $C, \delta>0$, independent of $u$, such that

$$
\|(u, v)\|_{C^{1, \alpha}\left(\Omega \backslash \Omega_{\delta}\right)} \leq C, \quad \text { for any } \quad \alpha \in(0,1) .
$$

Step 2. Let $\theta \in(0,1)$ be such that

$$
\frac{1}{p+1}=\theta \frac{N-2}{N} \quad \text { and } \quad \frac{1}{q+1}=(1-\theta) \frac{N-2}{N},
$$

Vol. 37, $\left.\mathrm{N}^{\circ} 1,2019\right]$ 
which is possible by (2).

Set $F(t):=\int_{0}^{t} f(s) d s \quad$ and $\quad G(t):=\int_{0}^{t} g(s) d s$, where

$$
f(s)=\frac{s^{p}}{[\ln (e+s)]^{\alpha}}, \quad g(s)=\frac{s^{q}}{[\ln (e+s)]^{\beta}} .
$$

Integrating by parts and taking into account (10) we have that

$$
F(t)-\theta\left(\frac{N-2}{N}\right) t f(t)=\frac{\alpha}{p+1} \int_{0}^{t} \frac{s^{p+1}}{\ln (e+s)^{\alpha+1}} \frac{d s}{e+s} .
$$

Likewise

$$
G(t)-(1-\theta)\left(\frac{N-2}{N}\right) t g(t)=\frac{\beta}{q+1} \int_{0}^{t} \frac{s^{q+1}}{\ln (e+s)^{\beta+1}} \frac{d s}{e+s} .
$$

Now, if we set $W(s, t):=F(t)+G(s)$, then $W_{s}=g(s)$ and $W_{t}=f(t)$. Therefore, for solutions $u>0$ and $v>0$ of (4),

$$
\begin{aligned}
\int_{\Omega}-[\Delta u(x \cdot \nabla v) & +\Delta v(x \cdot \nabla u)]=\int_{\Omega} \sum_{j} x_{j}\left(\frac{\partial W}{\partial v} \frac{\partial v_{j}}{\partial x_{j}}+\frac{\partial W}{\partial u} \frac{\partial u_{j}}{\partial x_{j}}\right) \\
& =\int_{\Omega} \sum_{j} x_{j} \frac{\partial W}{\partial x_{j}}=-N \int_{\Omega} W+\int_{\Omega} \operatorname{div}(W \vec{x}) \\
& =-N \int_{\Omega}[F(v)+G(u)]+\int_{\partial \Omega}(x \cdot n) W(u, v),
\end{aligned}
$$

and

$$
\int_{\Omega} \nabla u \nabla v=(1-\theta) \int_{\Omega} u g(u)+\theta \int_{\Omega} v f(v) .
$$

Applying Lemma 2.2 (Pohozaev-Rellich-Mitidieri type identity) we get that

$$
\begin{aligned}
N \int_{\Omega}[F(v)+G(u)]- & (N-2) \int_{\Omega}[\theta v f(v)+(1-\theta) u g(u)] \\
= & \int_{\partial \Omega}(x \cdot n) W(u, v)-\int_{\partial \Omega}(\nabla u \cdot \nabla v)(x \cdot n) \\
& +\int_{\partial \Omega} \frac{\partial u}{\partial n}(x \cdot \nabla v)+\int_{\partial \Omega} \frac{\partial v}{\partial n}(x \cdot \nabla u) .
\end{aligned}
$$

Therefore, it follows from (13) and (9) that

$$
\left|N \int_{\Omega}[F(v)+G(u)]-(N-2) \int_{\Omega}[\theta v f(v)+(1-\theta) u g(u)]\right| \leq C .
$$


Using (11), (12), and (14), we obtain that

$$
\begin{aligned}
\mid \frac{\alpha}{p+1} \int_{\Omega} & \left(\int_{0}^{v(x)} \frac{s^{p+1}}{\ln (e+s)^{\alpha+1}} \frac{d s}{e+s}\right) d x \\
& +\frac{\beta}{q+1} \int_{\Omega}\left(\int_{0}^{u(x)} \frac{s^{q+1}}{\ln (e+s)^{\beta+1}} \frac{d s}{e+s}\right) d x \mid \leq C .
\end{aligned}
$$

Moreover,

$$
\lim _{t \rightarrow \infty} \frac{\int_{0}^{t}\left(\frac{1}{\ln (e+s)}\right)^{\alpha+1} \frac{s^{p+1}}{e+s} d s}{\frac{t^{p+1}}{\ln (e+t)^{\alpha+1}}}=\frac{1}{p+1}
$$

and

$$
\lim _{t \rightarrow \infty} \frac{\int_{0}^{t}\left(\frac{1}{\ln (e+s)}\right)^{\beta+1} \frac{s^{q+1}}{e+s} d s}{\frac{t^{q+1}}{\ln (e+t)^{\beta+1}}}=\frac{1}{q+1} .
$$

Therefore, for any $\varepsilon>0$ there exists a constant $t_{\varepsilon}$, such that if $t>t_{\varepsilon}$, then

$$
\frac{1}{p+1}-\varepsilon<\frac{\int_{0}^{t}\left(\frac{1}{\ln (e+s)}\right)^{\alpha+1} \frac{s^{p+1}}{e+s} d s}{\frac{t^{p+1}}{\ln (e+t)^{\alpha+1}}}
$$

and

$$
\frac{1}{q+1}-\varepsilon<\frac{\int_{0}^{t}\left(\frac{1}{\ln (e+s)}\right)^{\beta+1} \frac{s^{q+1}}{e+s} d s}{\frac{t^{q+1}}{\ln (e+t)^{\beta+1}}}
$$

Let us choose $\varepsilon=\frac{1}{2} \min \left\{\frac{1}{p+1}, \frac{1}{q+1}\right\}$; then there exists a constant $C>0$ such that for any $t>0$,

$$
\frac{t^{p+1}}{\ln (e+t)^{\alpha+1}} \leq C\left(1+\int_{0}^{t}\left(\frac{1}{\ln (e+s)}\right)^{\alpha+1} \frac{s^{p+1}}{e+s} d s\right)
$$

and

$$
\frac{t^{q+1}}{\ln (e+t)^{\beta+1}} \leq C\left(1+\int_{0}^{t}\left(\frac{1}{\ln (e+s)}\right)^{\beta+1} \frac{s^{q+1}}{e+s} d s\right) .
$$

Vol. 37, $\left.\mathrm{N}^{\circ} 1,2019\right]$ 
Hence, applying the above inequalities for any $v(x)$ and $u(x)$ solving (4) respectively, integrating in $\Omega$ and using (15), we have that

$$
\left|\int_{\Omega} \frac{v^{p+1}}{\ln (e+v)^{\alpha+1}}+\int_{\Omega} \frac{u^{q+1}}{\ln (e+u)^{\beta+1}}\right| \leq C .
$$

This implies that

$$
\begin{gathered}
\int_{\Omega} \frac{v f(v)}{\ln (e+v)}=\int_{\Omega} \frac{v^{p+1}}{\ln (e+v)^{\alpha+1}} \leq C, \\
\int_{\Omega} \frac{u g(u)}{\ln (e+u)}=\int_{\Omega} \frac{u^{q+1}}{\ln (e+u)^{\beta+1}} \leq C .
\end{gathered}
$$

As pointed out in Remark 1.2, condition (5) ensures that $f$ and $g$ are nondecreasing.

Step 3. Now, let us fix $r, r^{\prime} \in\left(\frac{N}{2}, N\right)$ such that $r \geq 1+\frac{1}{p}$ and $r^{\prime} \geq 1+\frac{1}{q}$. Then using (16) and the fact that $f$ and $g$ are nondecreasing for $\|v\|_{\infty}$ large enough, we get that

$$
\begin{aligned}
\int_{\Omega}|f(v)|^{r} & =\int_{\Omega} \frac{v^{p r}}{\ln (e+v)^{\alpha r}} \\
& =\int_{\Omega} \frac{v^{p r-p-1}}{\ln (e+v)^{\alpha(r-1)-1}} \frac{v^{p+1}}{\ln (e+v)^{\alpha+1}} \\
& \leq C \frac{\|v\|_{\infty}^{p r-(p+1)}}{\ln \left(e+\|v\|_{\infty}\right)^{\alpha(r-1)-1}} \\
& =C\left(\frac{\|v\|_{\infty}^{p}}{\ln \left(e+\|v\|_{\infty}\right)^{\alpha}}\right)^{r-1-\frac{1}{p}} \frac{1}{\ln \left(e+\|v\|_{\infty}\right)^{\frac{\alpha}{p}-1}},
\end{aligned}
$$

and similarly

$$
\int_{\Omega}|g(u)|^{r^{\prime}} \leq C\left(\frac{\|u\|_{\infty}^{q}}{\ln \left(e+\|u\|_{\infty}\right)^{\beta}}\right)^{r^{\prime}-1-\frac{1}{q}} \frac{1}{\ln \left(e+\|u\|_{\infty}\right)^{\frac{\beta}{q}-1}} .
$$

All the other arguments work as in part I, Theorems 2.1, 2.2 (see also [71, proof of Theorems 1.1 and 1.3]).

Proof of Theorem 1.3. The proof is divided in two parts. We first prove that if there exists a positive solution $((\lambda, \mu),(u, v))$ of equation (6) with $\lambda, \mu \geq 0$, then $\lambda \mu<\lambda_{1}^{2}$. In the second part, we prove the converse.

Part I. Assume that there exists a positive solution $((\lambda, \mu),(u, v))$ of equation (6), and that $\lambda, \mu \geq 0$. Let $\phi_{1}>0$ be the principal eigenfunction associated to $\lambda_{1}$ and normalized in the $L^{2}(\Omega)$ norm. Multiplying each equation of (6) by $\phi_{1}$, and integrating by parts on $\Omega$, it yields that

$$
\begin{aligned}
\lambda_{1} \int_{\Omega} u \phi_{1} & =\lambda \int_{\Omega} v \phi_{1}+\int_{\Omega} f(v) \phi_{1}, \\
\lambda_{1} \int_{\Omega} v \phi_{1} & =\mu \int_{\Omega} u \phi_{1}+\int_{\Omega} g(u) \phi_{1} .
\end{aligned}
$$

[Revista Integración, temas de matemáticas 
Multiplying the first equation by $\lambda_{1}$, the second equation by $\lambda$ and adding both equations we deduce

$$
\left(\lambda_{1}^{2}-\lambda \mu\right) \int_{\Omega} u \phi_{1}=\int_{\Omega}\left[\lambda_{1} f(v)+\lambda g(u)\right] \phi_{1}>0 .
$$

Thus, $\lambda \mu<\lambda_{1}^{2}$.

Part II. Assume that $\lambda, \mu \geq 0$ and $\lambda \mu<\lambda_{1}^{2}$; we will prove that there is a positive solution $(u, v)$ of $(6)$. The proof is divided in three steps. In step 1, we reformulate problem (6) in abstract (operators) setting. In step 2 , we fix one parameter, say, $\lambda=\lambda_{0}>0$, and choosing $\mu$ as bifurcation parameter, we use Crandall-Rabinowitz's Theorem to prove that when $\sqrt{\lambda_{0} \mu}=\lambda_{1}$ there is a bifurcation phenomena from the trivial solution. Moreover, at least locally, the nontrivial solution pairs $(u, v)$ are strictly positive. In step 3 we use the global bifurcation result stated by Rabinowitz [82] and completed by Dancer [34] (see also [35], [63]) to prove that equation (6) has at least one solution. We conclude with a remark on the fact that varying $\lambda$ we obtain a whole curve of non-isolated bifurcation points, and using Alexander and Antman's result [3], we can deduce that in a neighborhood of that curve, there is a bifurcating two-dimensional surface of nontrivial solution pairs $((\lambda, \mu),(u, v))$ of equation $(6)$.

Step1. We start by reformulating problem (6).

Let $\bar{f}$ and $\bar{g}$ be the extension of $f$ and $g$, defined by

$$
\bar{f}(t)=\frac{|t|^{p}}{[\ln (e+|t|)]^{\alpha}}, \quad \bar{g}(s)=\frac{|s|^{q}}{[\ln (e+|s|)]^{\beta}}, \quad \text { for } \quad s, t \leq 0
$$

and denote by $F(w):=\left(\begin{array}{l}\bar{f}(v) \\ \bar{g}(u)\end{array}\right)$. Then (6) can be extended to non-positive and changing sign solutions and can be rewritten

$$
-\Delta w=A w+F(w), \quad \text { in } \Omega, \quad w=\left(\begin{array}{l}
0 \\
0
\end{array}\right) \quad \text { on } \partial \Omega,
$$

where $A:=\left(\begin{array}{cc}0 & \lambda \\ \mu & 0\end{array}\right), w:=\left(\begin{array}{c}u \\ v\end{array}\right)$, and any positive solution $(u, v)$ of $(17)$ is a positive solution of (6), and conversely.

Following the same ideas used in the proof of Theorem 1.1, it can be easily checked that for any $[a, b] \times[c, d] \subset \mathbb{R}_{+}^{2}$, there exists a constant $C>0$ such that any positive solution $(u, v)$ of equation (17) satisfies

$$
\|u\|_{L^{\infty}(\Omega)} \leq C, \quad\|v\|_{L^{\infty}(\Omega)} \leq C, \quad \forall(\lambda, \mu) \in[a, b] \times[c, d] .
$$

Assume that $\lambda, \mu>0$ and consider the Jordan canonical form of the matrix $A$. We can decompose $A=P^{-1} J P$, where

$$
J=\left(\begin{array}{cc}
\sqrt{\lambda \mu} & 0 \\
0 & -\sqrt{\lambda \mu}
\end{array}\right), P=\frac{1}{2}\left(\begin{array}{cc}
\sqrt{\frac{\mu}{\lambda}} & 1 \\
-\sqrt{\frac{\mu}{\lambda}} & 1
\end{array}\right), P^{-1}=\left(\begin{array}{cc}
\sqrt{\frac{\lambda}{\mu}} & -\sqrt{\frac{\lambda}{\mu}} \\
1 & 1
\end{array}\right) .
$$

Vol. 37, $\left.\mathrm{N}^{\circ} 1,2019\right]$ 
Multiplying (17) by $P$ on the left and denoting by $z=P w$, we obtain

$$
-\Delta z=J z+G(z), \quad \text { in } \Omega, \quad z=\left(\begin{array}{l}
0 \\
0
\end{array}\right) \quad \text { on } \partial \Omega,
$$

where $G(z):=P F(w)=P F\left(P^{-1} z\right)$.

Step 2. We check that the conditions of Crandall-Rabinowitz's Theorem [24] are satisfied. Fix $\lambda=\lambda_{0}>0$, and choose $\mu$ as the bifurcation parameter. Let $\sigma \in(0,1)$; define $E_{2}=\left\{u \in C^{2, \sigma}(\bar{\Omega}): u=0\right.$ on $\left.\partial \Omega\right\}$ equipped with its standard norm; $E_{2}$ is a Banach space. Set $E_{0}=C^{\sigma}(\bar{\Omega})$ and define the operator $\mathbf{F}: \mathbb{R} \times\left(E_{2}\right)^{2} \rightarrow\left(C^{\sigma}(\bar{\Omega})\right)^{2}$ by

$$
\begin{aligned}
\mathbf{F}(\mu, w) & :=-\Delta w-\left(\begin{array}{cc}
0 & \lambda_{0} \\
\mu & 0
\end{array}\right) w-F(w) ; \\
L_{0} w & :=D_{(u, v)} \mathbf{F}\left(\mu_{0}, 0\right) w=-\Delta w-\left(\begin{array}{cc}
0 & \lambda_{0} \\
\mu_{0} & 0
\end{array}\right) w \\
L_{1} w & :=D_{\mu,(u, v)} \mathbf{F}\left(\mu_{0}, 0\right) w=\left(\begin{array}{c}
0 \\
-v
\end{array}\right), \quad \text { where } \quad w=\left(\begin{array}{c}
u \\
v
\end{array}\right) .
\end{aligned}
$$

Set $\mu_{0}=\frac{\lambda_{1}^{2}}{\lambda_{0}}$, and $P_{0}=P\left(\lambda_{0}, \mu_{0}\right)$. Observe that $w \in N\left(L_{0}\right)$ (where $N\left(L_{0}\right)$ is the kernel of $\left.L_{0}\right)$ if, and only if, $z=P_{0} w \in N\left(-\Delta-\left(\begin{array}{cc}\lambda_{1} & 0 \\ 0 & -\lambda_{1}\end{array}\right)\right)=\operatorname{span}\left[\left(\begin{array}{c}\phi_{1} \\ 0\end{array}\right)\right]$. Therefore, $N\left(L_{0}\right)=\operatorname{span}\left[\left(\begin{array}{c}\lambda_{0} \phi_{1} \\ \lambda_{1} \phi_{1}\end{array}\right)\right]$.

Now, we claim that $L_{1}\left(N\left(L_{0}\right)\right) \not \subset R\left(L_{0}\right)$, where $R\left(L_{0}\right)$ is the range of $L_{0}$.

Indeed, assume that there exist $w \in N\left(L_{0}\right)$ and $\psi=\left(\begin{array}{c}\psi_{1} \\ \psi_{2}\end{array}\right) \in D\left(L_{0}\right)$ such that $L_{1} w=L_{0} \psi$ or, equivalently by definition of $L_{0}$ and $L_{1}$,

$$
-\Delta \psi-\left(\begin{array}{cc}
0 & \lambda_{0} \\
\mu_{0} & 0
\end{array}\right) \psi=a\left(\begin{array}{c}
0 \\
-\lambda_{1} \phi_{1}
\end{array}\right), \quad \text { for some } a \in \mathbb{R} .
$$

Multiplying (19) on the left by $P_{0}$, and denoting by $\varphi=P_{0} \psi=\left(\begin{array}{c}\varphi_{1} \\ \varphi_{2}\end{array}\right)$, we obtain

$$
-\Delta \varphi-\left(\begin{array}{cc}
\lambda_{1} & 0 \\
0 & -\lambda_{1}
\end{array}\right) \varphi=-\frac{a \lambda_{1}}{2}\left(\begin{array}{c}
\phi_{1} \\
\phi_{1}
\end{array}\right) .
$$

Multiplying the first (component) equation by $\phi_{1}$, integrating on $\Omega$, and applying Green's formulae we obtain

$$
0=\int_{\Omega}\left(-\Delta \varphi_{1}-\lambda_{1} \varphi_{1}\right) \phi_{1}=-\frac{a \lambda_{1}}{2} \int_{\Omega} \phi_{1}^{2} .
$$

Therefore $a=0$. Hence, the hypotheses of Crandall Rabinowitz theorem are satisfied. Thus, there exists a neighborhood of $\left(\frac{\lambda_{1}^{2}}{\lambda_{0}},(0,0)\right)$ in $\mathbb{R} \times\left(E_{2}\right)^{2}$, and continuous functions 
$\mu(s), \tilde{w}(s), s \in(-\epsilon, \epsilon)$, such that $\mu(0)=\mu_{0}, \tilde{w}(0)=\left(\begin{array}{l}0 \\ 0\end{array}\right), \tilde{w}=\left(\begin{array}{c}\tilde{w}_{1} \\ \tilde{w}_{2}\end{array}\right)$, with $\int_{\Omega} \tilde{w}_{i} \phi_{1}=0$, and the only nontrivial solutions of (6) for $\lambda=\lambda_{0}$ fixed, are

$$
\left(\mu(s), s\left(\begin{array}{c}
\lambda_{0} \phi_{1} \\
\lambda_{1} \phi_{1}
\end{array}\right)+s \tilde{w}(s)\right) .
$$

Observe that for $s>0$ small enough, $w=\left(\begin{array}{c}u \\ v\end{array}\right)$ satisfies $u>0, v>0, \frac{\partial u}{\partial n}<0, \frac{\partial v}{\partial n}<0$ on $\partial \Omega$, hence $\lambda_{0} \mu<\lambda_{1}^{2}$.

Step 3. Now, we use the global bifurcation Theorem as stated by Rabinowitz [82] and as completed by Dancer [34]. Let $(-\Delta)^{-1}$ denote the inverse of $(-\Delta)$ with homogeneous Dirichlet boundary conditions. It follows from Schauder estimates that $(-\Delta)^{-1}$ maps bounded subsets of $E_{0}$ into bounded subsets of $E_{2}$, which in turn are relatively compact in $E_{0}$. Thus, $(-\Delta)^{-1}: E_{0} \rightarrow E_{0}$ is compact.

Observe that, fixed points of the operator $(-\Delta)^{-1}[J()+.G()$.$] corresponds to fixed points$ of the operator $(-\Delta)^{-1}[A()+.F()$.$] , that is,$

$$
z=(-\Delta)^{-1}[J z+G(z)] \Longleftrightarrow w=(-\Delta)^{-1}[A w+F(w)]
$$

Let us keep fixed $\lambda=\lambda_{0}>0$, and allow $\mu$ to vary. It follows from Rabinowitz's global bifurcation Theorem [82, Theorem 1.3] that there is a continuum of solutions, emanating from the trivial solution at $(\lambda, \mu)=\left(\lambda_{0}, \frac{\lambda_{1}^{2}}{\lambda_{0}}\right)$, which is either unbounded, or meets another bifurcation point from the trivial solution. Let

$$
\mathcal{C}_{\lambda_{0}}:=\left\{\left(\left(\lambda_{0}, \mu\right),\left(u_{\lambda_{0}, \mu}, v_{\lambda_{0}, \mu}\right)\right) \in \mathbb{R}^{2} \times\left(C^{\sigma}(\bar{\Omega})\right)^{2}\right\}
$$

be the continuum emanating from the trivial solution at $\mu=\mu_{0}=\frac{\lambda_{1}^{2}}{\lambda_{0}}$, and solving (6) for $\lambda=\lambda_{0}$ fixed. By elliptic regularity, it is known that $\mathcal{C}_{\lambda_{0}} \subset \mathbb{R}^{2} \times\left(C^{2, \sigma}(\bar{\Omega})\right)^{2}$.

Considering the positive cone $\mathcal{P}:=\left\{u \in C^{1, \sigma}(\bar{\Omega}): u>0\right.$, in $\Omega, \frac{\partial u}{\partial n}<0$, on $\left.\partial \Omega\right\}$, let us denote by $\mathcal{C}_{\lambda_{0}}^{+}:=\mathcal{C}_{\lambda_{0}} \cap \mathbb{R}^{2} \times(\mathcal{P})^{2} \neq \emptyset$. Since the classical positive solutions are a priori bounded (see (18)), we have that $\mathcal{C}_{\lambda_{0}}^{+} \cap\left(\left\{\lambda_{0}\right\} \times\left[0, \frac{\lambda_{1}^{2}}{\lambda_{0}}\right) \times\left(C^{\sigma}(\bar{\Omega})\right)^{2}\right)$ is bounded. Assume that there exists $\mu=\mu^{*} \in\left[0, \frac{\lambda_{1}^{2}}{\lambda_{0}}\right)$ such that $\left(\left(\lambda_{0}, \mu^{*}\right),\left(u^{*}, v^{*}\right)\right) \in \overline{\mathcal{C}_{\lambda_{0}}^{+}} \backslash \mathcal{C}_{\lambda_{0}}^{+}$; then, either $\left(u^{*}, v^{*}\right)=(0,0)$ or $u^{*} \geq 0, v^{*} \geq 0$ in $\Omega, \frac{\partial u^{*}}{\partial n} \leq 0, \frac{\partial v^{*}}{\partial n} \leq 0$ on $\partial \Omega$, with $\left(u^{*}, v^{*}\right) \neq(0,0)$. If $\left(u^{*}, v^{*}\right)=(0,0)$ then $\left(\left(\lambda_{0}, \mu^{*}\right),\left(u^{*}, v^{*}\right)\right)$ is a bifurcation point from the trivial solution to positive solutions. Due to the unique bifurcation point from the trivial solution to positive solutions at $\lambda=\lambda_{0}$ is attained at $\mu=\frac{\lambda_{1}^{2}}{\lambda_{0}}$, if $\left(u^{*}, v^{*}\right)=(0,0)$ then we reach a contradiction. On the other hand, if $u^{*} \geq 0, v^{*} \geq 0$ in $\Omega,\left(u^{*}, v^{*}\right) \neq(0,0)$, from the Maximum Principle and the Hopf Maximum Principle $u^{*}>0, v^{*}>0$ in $\Omega$, and $\frac{\partial u^{*}}{\partial n^{*}}<0$, $\frac{\partial v}{\partial n}<0$; therefore $\left(\left(\lambda_{0}, \mu^{*}\right),\left(u^{*}, v^{*}\right)\right) \in \mathcal{C}_{\lambda_{0}}^{+}$, which contradicts the hypothesis.

Remark 2.3. Let us mention that when moving $\lambda$ we obtain a whole curve of nonisolated bifurcation points. If $\mathcal{S}$ denote the closure of the set of nontrivial solutions pairs

Vol. 37, $\mathrm{N}^{\circ}$ 1, 2019] 
$((\lambda, \mu), w)$ of $(17)$, and $\mathcal{F}$ denote the set of bifurcation points of (17) from the trivial solution, we proved in Step 2 that the set

$$
\mathcal{F}_{1}:=\left\{\left(\left(\lambda, \frac{\lambda_{1}^{2}}{\lambda}\right),(0,0)\right): \lambda>0\right\}
$$

is a set of bifurcation points of (17) from the trivial solution. All points in $\mathcal{F}_{1}$ are nonisolated bifurcation points. Using Alexander and Antman's result [3], we can deduce that in a neighborhood of that curve, there is a bifurcating two-dimensional surface of nontrivial solution pairs $((\lambda, \mu),(u, v))$ of equation $(6)$.

\section{A priori estimates for quasilinear elliptic equations involving the p-Laplacian}

Let $\Omega$ be a smooth, bounded, and strictly convex domain in $\mathbb{R}^{N}, N \geq 2$. We prove $L^{\infty}(\Omega)$ a priori bounds for $C^{1}(\bar{\Omega})$ weak solutions of the problem

$$
\left\{\begin{aligned}
-\Delta_{p}(u)=f(u) & \text { in } \Omega, \\
u>0 & \text { in } \Omega, \\
u=0 & \text { on } \partial \Omega,
\end{aligned}\right.
$$

where $\Delta_{p}(u)=\operatorname{div}\left(|D u|^{p-2} D u\right)$ is the $p$-Laplace operator, $1<p<\infty$, and

$\left(H_{1}\right) \quad f:[0, \infty) \rightarrow \mathbb{R}$ is a locally Lipschitz continuous function with $f(0) \geq 0$.

If $p>2$ we also assume that $f(s)>0$ if $s>0$.

The equation $-\Delta_{p} u=f(u)$ is the $L^{p}$ counterpart to the classical semilinear elliptic equation $-\Delta u=f(u)$, and appears for instance in the theory of non-Newtonian fluids, dilatant fluids in the case $p \geq 2$, pseudo-plastic fluids in the case $1<p<2$ (see [6], [69], [70]).

If $u \in W^{1, p}(\Omega) \cap L^{\infty}(\Omega)$ is a weak solution of the problem $(20)$, then $u \in C^{1, \tau}(\bar{\Omega})$ with $\tau<1$ (see [41], [60]), so we assume from the beginning a $C^{1}$ regularity for the solution, which is in general only a weak solution. A standard setting in the applications of $a$ priori estimates to existence of solutions to elliptic problems, is the space of continuous functions. If $u \in C^{0}(\bar{\Omega})$, then also $f(u)$ is continuous and the solution $u$ belongs to the space $C^{1, \tau}(\bar{\Omega})$, by the cited regularity results.

Mitidieri and Pohozaev in [73], [74] proved Liouville theorems for quasilinear elliptic inequalities in $\mathbb{R}^{N}$ involving the $p$-laplacian. Later, Serrin and Zou [86], and also Farina and Serrin [42], [43], proved Liouville theorems for more general operators.

With the help of the blow-up procedure, Azizieh and Clement in [9] proved a priori estimates for the equation (20) when $\Omega$ is strictly convex, $1<p<2$ and $f(u)$ growing not faster than a power $u^{\vartheta}$ at infinity, with

$$
1<\vartheta<p_{*}-1, \quad p_{*}=\frac{(N-1) p}{N-p}
$$

[Revista Integración, temas de matemáticas 
(see [10] for the case of systems). The exponent $p_{*}=\frac{(N-1) p}{N-p}$ is the optimal exponent for Liouville theorems for elliptic inequalities; observe that

$$
p_{*}=\frac{(N-1) p}{N-p}<p^{*}=\frac{N p}{N-p},
$$

where $p^{*}$ is the critical exponent for the Sobolev's embeddings. The restriction $1<p<2$ depends on the fact that using a blow-up procedure and Liouville theorems on the whole space, they need to exclude concentration of maximum points of the solutions at the boundary, and they use some result proved in [29], [30] on the symmetry and monotonicity of solutions to $p$-Laplace equations in the singular case $1<p<2$; these results were later extended to the case $p>2$ in the papers [32], [33].

Ruiz [83] proved a priori estimates for equation more general than (20), using a different technique based among other tools on Harnack type inequalities; therein $f=f(x, u, D u)$ can depend on $x$ and on the gradient, for any $1<p<N$ and for general domains. Once again the growth at infinity with respect to $u$ must be less than powers with exponent $\vartheta<p_{*}-1$.

In both papers, there is also a general discussion on how the existence of solutions follows from the a priori estimates, using some abstract results by Krasnoselskij already used in [40].

Later, Zou [92] proved Liouville theorems in half spaces that, together with the results in [84], allow him to use the blow-up method and prove a priori estimates for equations more general than (20), in case $1<p<N$; therein $f=f(x, u, D u)$ can depend on $x$ and on the gradient, and under various hypotheses on the nonlinearities. In particular, it is assumed that $f=f(x, u, D u)$ grows with respect to $u$ as a subcritical power at infinity and zero.

For monotonicity and Liouville type theorems in half spaces many other papers appeared recently (see in particular the papers of Farina, Montoro, and Sciunzi [44], [45], [46], and Farina, Montoro, Riey, and Sciunzi [47]).

In recent years, related a priori estimates for general operators were established by D'Ambrosio and Mitidieri in [26], [27].

The aim of this Section is to state a priori estimates for solutions of (20) in the case of $\Omega$ being a smooth bounded strictly convex domain, for any value of $p>1$. In case $1<p<N, f(u)$ is assumed to have a subcritical grow at infinity, but allowing more general functions than merely subcritical powers (see for example Corollary 1.4).

We adapt the technique introduced in [40] for the case $p=2$, that allows to give the same proof in cases $1<p<N, p=N$ and $p>N$. Of course, in the latter cases, much weaker hypotheses are needed in order to obtain the desired estimates (in particular in case $p>N$ we only need that $f(u)$ grows faster than $u^{p-1}$ at infinity, condition that for $p=2$ is the superlinearity at infinity). This proofs rely deeply on the $C^{2}$ regularity of solutions, which are then classical solutions, and on the $W^{2, q}$ estimates based on the Calderón-Zygmund and Agmon-Douglis-Nirenberg estimates [1], [2]. These estimates are not available in the singular/degenerate case $p \neq 2$. We use instead regularity results for the $p$-laplacian (see [41], [60], [88], and gradient estimates [57], [19], [20]). There are many other tools

Vol. 37, $\left.\mathrm{N}^{\circ} 1,2019\right]$ 
that we had to adapt to handle in our case. We use Strong Maximum Principle and Hopf's Lemma for the $p$-Laplacian (see [91]), weak and strong comparison principles for the $p$-Laplacian (see [25], [28], [32], [33], [55], [78]), Picone's identity for the $p$-Laplacian ([4]), Pohozaev's identity for the $p$-Laplacian ([55]). Although the general theory of eigenvalues for the $p$-Laplacian is far from complete (see [50], [77]), the properties of the first eigenvalue are known and are the same as in the case $p=2$ (see [5], [77], [61]).

All the following results are already known (see [31]):

Theorem 3.1 (Case $p>N$ ). Let $\Omega$ be a smooth bounded domain in $\mathbb{R}^{N}, N \geq 2$, which is strictly convex. Assume that $p>N$, the condition $\left(H_{1}\right)$ holds and

$\left(H_{2}\right) \quad$ there exist $\tau>0$ and $C_{1}>0$ such that

$\liminf _{s \rightarrow+\infty} \frac{f(s)}{s^{p-1+\tau}}>C_{1}>0$.

Then, the solutions of (20) are a priori bounded in $L^{\infty}$ : there exists a constant $C$, depending on $p, \Omega$ and $f$, but independent of the solution $u$, such that $\|u\|_{L^{\infty}(\Omega)} \leq C$ for any solution of $(20)$.

Proof. See [31, Proof of Theorem 1.1] for a proof.

Remark 3.2. For the ordinary laplacian $(p=2)$, the above theorem corresponds to the case of dimension $N=1$. In [40, Remark 1.3] it was observed that if $N=1$, solutions are uniformly bounded under the only hypothesis of superlinearity at infinity, which corresponds for $p=2$ to the hypothesis $\left(H_{2}\right)$ with $\tau=0$, and the bound from below strictly bigger than $\lambda_{1}$, the first eigenvalue for the Laplacian operator.

We need the slightly stronger form (with $\tau>0$ but arbitrarily small) for technical reasons (use of the Picone's Identity for the $p$-laplacian).

Theorem 3.3 (Case $p=N$ ). Let $\Omega$ be a smooth bounded domain in $\mathbb{R}^{N}, N \geq 2$, which is strictly convex. Let $p=N$ and assume that $\left(H_{1}\right)$ and $\left(H_{2}\right)$ hold, as well as

$\left(H_{3}\right)$ there exists $C_{2}>0$ such that $\liminf _{s \rightarrow+\infty} \frac{F(s)}{s f(s)}>C_{2}>0$, where $F(t)=\int_{0}^{t} f(s) d s$ is a primitive of the function $f$;

$\left(H_{4}\right)$ there exists $\theta>0$ such that $\limsup _{s \rightarrow+\infty} \frac{|f(s)|}{s^{\theta}}<+\infty$ (of course this is equivalent to: there exists $\eta>0$ such that $\left.\lim _{s \rightarrow+\infty} \frac{|f(s)|}{s^{\eta}}=0\right)$.

Then, the solutions of (20) are a priori bounded in $L^{\infty}$ : there exists a constant $C$, depending on $p, \Omega$ and $f$, but independent of the solution $u$, such that $\|u\|_{L^{\infty}(\Omega)} \leq C$ for any solution of $(20)$.

Proof. See [31, Proof of Theorem 1.3] for a proof. 
Remark 3.4. If $p=2$ (the ordinary laplacian), the above theorem corresponds to the case of dimension $N=2$, and in that case solutions are uniformly bounded under the only hypotheses of superlinearity together with polynomial growth at infinity (cf. [40, Theorem 1.1]). All the functions $f$ growing polynomially at infinity are included in hypotheses $\left(H_{3}\right)$, and $\left(H_{4}\right)$.

Nevertheless, when $p=N$ the critical embedding is of exponential type, and those hypotheses are not optimal (neither are the hypotheses in [40]), and we think that they can be improved.

The following result can be seen as the counterpart for $p \neq 2$ to the results above (see [31, Proof of Theorem 1.7] for a proof).

Theorem 3.5 (Case $1<p<N$ ). Let $\Omega$ be a smooth bounded domain in $\mathbb{R}^{N}, N \geq 2$, which is strictly convex.

Let $1<p<N$, and assume that $\left(H_{1}\right)$ and $\left(H_{2}\right)$ hold, as well as

$\left(H_{3}\right.$ ") there exist a nonincreasing positive function $H:[0,+\infty) \rightarrow \mathbb{R}$ such that

$$
\liminf _{s \rightarrow+\infty} \frac{p^{*} F(s)-s f(s)}{s f(s) H(s)}>0,
$$

where $p^{*}=\frac{N p}{N-p}$, and

$\left(H_{4} "\right) \lim _{s \rightarrow+\infty} \frac{f(s)}{s^{p^{*}-1}[H(s)]^{\frac{p}{N-p}}}=0 ;$

$\left(H_{5}\right)$ there exist $C_{4}>0, C_{5}>0$ such that

$$
\liminf _{s \rightarrow+\infty} \frac{\min _{\left[\frac{s}{2}, s\right]} f}{f(s)} \geq C_{4}>0, \quad \limsup _{s \rightarrow+\infty} \frac{\max _{[0, s]} f}{f(s)} \leq C_{5} .
$$

Then, the solutions of (20) are a priori bounded in $L^{\infty}$ : there exists a constant $C$, depending on $p, \Omega$ and the function $f$, but independent of the solution $u$, such that $\|u\|_{L^{\infty}(\Omega)} \leq C$ for any solution of $(20)$.

The existence of solutions for (20) follows from the a priori estimates, with a further hypothesis about the behavior of the nonlinearity at zero.

This was proved in [40] (with the hypothesis $\left(H_{0}\right)$ below) for $p=2$, using some variants of topological arguments, connected with theorems of Krasnoselskii [58] and Rabinowitz [81] based on degree theory. It was extended to the case of $p$-Laplace equations in [9], [83], [92].

It also can be adapted to our hypotheses. More precisely, the following result holds (see [31, Proof of Theorem 1.8] for a proof).

Theorem 3.6. Let us assume that the hypotheses of one of the previous theorem hold, and assume also that

Vol. 37, $\mathrm{N}^{\circ}$ 1, 2019] 
$\left(H_{0}\right) \quad \limsup _{s \rightarrow 0^{+}} \frac{f(s)}{s^{p-1}}<\lambda_{1, p}$,

where $\lambda_{1, p}$ is the first eigenvalue for the $p$-Laplacian (see [31, Section 2]). (Since $f(0) \geq 0$ by $\left(H_{1}\right)$, this hypothesis implies that $\left.f(0)=0\right)$.

Then, there exists a positive solution of (20).

\section{Equivalence between uniform $L^{2^{\star}}(\Omega)$ a-priori bounds and uniform $L^{\infty}(\Omega)$ a-priori bounds for subcritical elliptic equations}

We consider the existence of $L^{\infty}(\Omega)$ a-priori bounds for classical positive solutions to the boundary value problem

$$
-\Delta u=f(u), \quad \text { in } \Omega, \quad u=0, \quad \text { on } \partial \Omega,
$$

where $\Omega \subset \mathbb{R}^{N}, N>2$, is a bounded domain with $C^{2}$ boundary $\partial \Omega$. Trudinger [90] proved that any weak solution in $H^{1}(\Omega)$ is in fact in $L^{\infty}(\Omega)$ and consequently in $C^{\infty}(\Omega)$.

We provide sufficient conditions on $f$ for uniform $L^{2^{*}}(\Omega)$ a-priori bounds to imply uniform $L^{\infty}(\Omega)$ a-priori bounds, where $2^{*}=\frac{2 N}{N-2}$ is the critical Sobolev exponent. The converse is obviously true without any additional hypotheses.

The ideas for the proof of the following theorem are similar to those used in [16, Theorem 1.1] (see also part I, Theorems 2.1, 2.2). Unlike the proof in [16], here we do not use Pohozaev or moving planes arguments.

Our main result is the following theorem.

Theorem 4.1. Assume that the nonlinearity $f: \mathbb{R}^{+} \rightarrow \mathbb{R}$ is a locally Lipschitzian function that satisfies:

(H1) There exists a constant $C_{0}>0$ such that $\liminf _{s \rightarrow \infty} \frac{\min _{[s / 2, s]} f}{f(s)} \geq C_{0}$.

(H2) There exists a constant $C_{1}>0$ such that $\limsup _{s \rightarrow \infty} \frac{\max _{[0, s]} f}{f(s)} \leq C_{1}$.

(F) $\lim _{s \rightarrow+\infty} \frac{f(s)}{s^{2^{\star}-1}}=0$; that is, $f$ is subcritical.

Then the following conditions are equivalent:

(i) there exists a uniform constant $C$ (depending only on $\Omega$ and $f$ ) such that for every positive classical solution $u$ of (21),

$$
\|u\|_{L^{\infty}(\Omega)} \leq C
$$

[Revista Integración, temas de matemáticas 
(ii) there exists a uniform constant $C$ (depending only on $\Omega$ and $f$ ) such that for every positive classical solution $u$ of (21),

$$
\int_{\Omega}|f(u)|^{\frac{2 N}{N+2}} d x \leq C
$$

(iii) there exists a uniform constant $C$ (depending only on $\Omega$ and $f$ ) such that for every positive classical solution $u$ of (21),

$$
\|u\|_{L^{2^{*}}(\Omega)} \leq C .
$$

Proof. All the arguments work in a similar way as in part I, Theorems 2.1, 2.1 (see also [15, proof of Theorem 1.1]).

In this Section, we also provide sufficient conditions for the equivalence of the existence of a uniform $L^{2^{\star}}(\Omega)$ a priori bound with that of a uniform $L^{\infty}(\Omega)$ a priori bound for sequences of boundary value problems. In fact, we have the following theorem, which is [15, Theorem 1.2]; we include the proof by the sake of completeness.

Theorem 4.2. Consider the following sequence of BVPs

$$
-\Delta v=g_{k}(v) \quad \text { in } \Omega, \quad v=0 \quad \text { on } \partial \Omega,
$$

with $g_{k}: \mathbb{R}^{+} \rightarrow \mathbb{R}$ locally Lipschitzian. We assume that the following hypotheses are satisfied:

$(\mathrm{H} 1)_{k}$ there exists a uniform constant $C_{1}>0$ such that $\liminf _{s \rightarrow+\infty} \frac{\min _{[s / 2, s]} g_{k}}{g_{k}(s)} \geq C_{1}$;

$(\mathrm{H} 2)_{k}$ there exists a uniform constant $C_{2}>0$ such that $\limsup _{s \rightarrow+\infty} \frac{\max _{[0, s]} g_{k}}{g_{k}(s)} \leq C_{2}$.

Let $\left\{v_{k}\right\}$ be a sequence of classical positive solutions to (21) $)_{k}$ for $k \in \mathcal{N}$. If

$(\mathrm{F})_{k} \lim _{k \rightarrow+\infty} \frac{g_{k}\left(\left\|v_{k}\right\|\right)}{\left\|v_{k}\right\|^{2 *}-1}=0$,

then, the following two conditions are equivalent:

(i) there exists a uniform constant $C$, depending only on $\Omega$ and the sequence $\left\{g_{k}\right\}$, but independent of $k$, such that for every $v_{k}>0$, classical solution to (21) ${ }_{k}$,

$$
\limsup _{k \rightarrow \infty}\left\|v_{k}\right\|_{L^{\infty}(\Omega)} \leq C
$$

(ii) there exists a uniform constant $C_{0}$, depending only on $\Omega$ and the sequence $\left\{g_{k}\right\}$, but independent of $k$, such that for every $v_{k}>0$, classical solution to (21) ${ }_{k}$,

$$
\limsup _{k \rightarrow \infty} \int_{\Omega}\left|g_{k}\left(v_{k}\right)\right|^{\frac{2 N}{N+2}} d x \leq C_{0}
$$

Vol. 37, $\left.\mathrm{N}^{\circ} 1,2019\right]$ 
(iii) there exists a uniform constant $C$ (depending only on $\Omega$ and the sequence $\left\{g_{k}\right\}$ ) such that for every positive classical solution $v_{k}$ of $(21)_{k}$,

$$
\left\|v_{k}\right\|_{L^{2^{*}}(\Omega)} \leq C .
$$

Hypothesis $(\mathrm{H} 1)_{k}$, and $(\mathrm{H} 2)_{k}$, are not sufficient for the existence of a uniform $L^{\infty}$ a priori bound. Atkinson and Peletier in [8] show that for $f_{\varepsilon}(s)=s^{2^{\star}-1-\varepsilon}$ and $\Omega$ a ball in $\mathbb{R}^{3}$, there exists $x_{0} \in \Omega$ and a sequence of solutions $u_{\varepsilon}$ such that $\lim _{\varepsilon \rightarrow 0} u_{\varepsilon}=0$ in $C^{1}\left(\Omega \backslash\left\{x_{0}\right\}\right)$ and $\lim _{\varepsilon \rightarrow 0} u_{\varepsilon}\left(x_{0}\right)=+\infty$. See also Han [56], for minimum energy solutions in general domains.

Furthermore, hypotheses $(\mathrm{H} 1)_{k},(\mathrm{H} 2)_{k}$, and $(\mathrm{F})_{k}$, are not sufficient for the existence of an $L^{\infty}$ a priori bound. In fact, at the end of this Section, we construct a sequence of BVP satisfying $(\mathrm{H} 1)_{k},(\mathrm{H} 2)_{k}$, and $(\mathrm{F})_{k}$, and a sequence of solutions $v_{k}$ such that $\lim _{k \rightarrow \infty}\left\|v_{k}\right\|_{\infty}=+\infty$. Our example also shows the non-uniqueness of positive solutions.

Remark 4.3. One can easily see that condition (22), elliptic regularity and Sobolev embeddings imply that there exists a uniform constant $C_{4}>0$ such that

$$
\limsup _{k \rightarrow \infty} \int_{\Omega} v_{k} g_{k}\left(v_{k}\right) d x \leq C_{4},
$$

for all classical positive solutions $\left\{v_{k}\right\}$ to $(21)_{k}$.

The ideas for the proof of the above theorem are similar to those used in [16, Theorem 1.1] (see also Theorems 2.1, 2.1 in part I). But, unlike the proof in [16], here we do not use Pohozaev or moving planes arguments; therefore, the structure of the proof described in Subsection 1.1 of part I will start in one adaptation of Step 3.

Proof of Theorem 4.2. Clearly, condition (i) implies (ii) and (iii). By the elliptic regularity and condition (22), we have that $\left\|v_{k}\right\|_{W^{2, \frac{2 N}{N+2}}} \leq C$. Therefore, $\left\|v_{k}\right\|_{H^{1}(\Omega)} \leq C$. So, by the Sobolev embedding, we deduce that

$$
\left\|v_{k}\right\|_{L^{2^{*}}(\Omega)} \leq C \quad \text { for all } k .
$$

Using similar arguments as in Theorems 2.1, 2.1 of part I and condition $(\mathrm{F})_{\mathrm{k}}$, one can show that (ii) and (iii) are equivalent. We shall concentrate our attention in proving that (ii) implies (i). All throughout this proof $C$ denotes several constants independent of $k$.

Observe that $1+\frac{1}{2^{\star}-1}=\frac{2 N}{N+2}$. From hypothesis (ii) (see (22)), there exists a fixed constant $C>0$ (independent of $k$ ) such that

$$
\begin{aligned}
\int_{\Omega}\left|g_{k}\left(v_{k}(x)\right)\right|^{q} d x & \leq \int_{\Omega}\left|g_{k}\left(v_{k}(x)\right)\right|^{1+\frac{1}{2^{\star}-1}}\left|g_{k}\left(v_{k}(x)\right)\right|^{q-1-\frac{1}{2^{\star}-1}} d x \\
& \leq C\left\|g_{k}\left(v_{k}(\cdot)\right)\right\|_{\infty}^{q-1-\frac{1}{2^{\star}-1}},
\end{aligned}
$$

for $k$ big enough, and for any $q>N / 2$.

[Revista Integración, temas de matemáticas 
Therefore, from elliptic regularity, (see [54, Lemma 9.17]),

$$
\left\|v_{k}\right\|_{W^{2, q}(\Omega)} \leq C\left\|\Delta v_{k}\right\|_{L^{q}(\Omega)} \leq C\left\|g_{k}\left(v_{k}(\cdot)\right)\right\|_{\infty}^{1-\frac{1}{q}-\frac{1}{\left(2^{\star}-1\right) q}}
$$

for $k$ big enough.

Let us restrict $q \in(N / 2, N)$. From Sobolev embeddings, for $1 / q^{*}=1 / q-1 / N$ with $q^{*}>N$, we can write

$$
\left\|v_{k}\right\|_{W^{1, q^{*}}(\Omega)} \leq C\left\|v_{k}\right\|_{W^{2, q}(\Omega)} \leq C\left\|g_{k}\left(v_{k}(\cdot)\right)\right\|_{\infty}^{1-\frac{1}{q}-\frac{1}{\left(2^{\star}-1\right) q}},
$$

for $k$ big enough.

From Morrey's Theorem (see [13, Theorem 9.12 and Corollary 9.14]), there exists a constant $C$ only dependent on $\Omega, q$ and $N$ such that

$$
\left|v_{k}\left(x_{1}\right)-v_{k}\left(x_{2}\right)\right| \leq C\left|x_{1}-x_{2}\right|^{1-N / q^{*}}\left\|v_{k}\right\|_{W^{1, q^{*}}(\Omega)}, \quad \forall x_{1}, x_{2} \in \Omega,
$$

for any $k$.

Therefore, for all $x \in B\left(x_{1}, R\right) \subset \Omega$,

$$
\left|v_{k}(x)-v_{k}\left(x_{1}\right)\right| \leq C R^{2-\frac{N}{q}}\left\|v_{k}\right\|_{W^{2, q}(\Omega)},
$$

for any $k$.

From now on, we argue by contradiction. Let $\left\{v_{k}\right\}$ be a sequence of classical positive solutions to $(21)_{k}$ and assume that

$$
\lim _{k \rightarrow \infty}\left\|v_{k}\right\|=+\infty, \quad \text { where } \quad\left\|v_{k}\right\|:=\left\|v_{k}\right\|_{\infty} .
$$

Let $x_{k} \in \Omega$ be such that

$$
v_{k}\left(x_{k}\right)=\max _{\Omega} v_{k}
$$

Let us choose $R_{k}$ such that $B_{k}:=B\left(x_{k}, R_{k}\right) \subset \Omega$, and

$$
v_{k}(x) \geq \frac{1}{2}\left\|v_{k}\right\| \quad \text { for any } \quad x \in B_{k},
$$

and there exists $y_{k} \in \partial B_{k}$ such that

$$
v_{k}\left(y_{k}\right)=\frac{1}{2}\left\|v_{k}\right\|
$$

Let us denote by

$$
m_{k}:=\min _{\left[\left\|v_{k}\right\| / 2,\left\|v_{k}\right\|\right]} g_{k}, \quad M_{k}:=\max _{\left[0,\left\|v_{k}\right\|\right]} g_{k} .
$$

Therefore, we obtain

$$
m_{k} \leq g_{k}\left(v_{k}(x)\right) \quad \text { if } x \in B_{k}, \quad g_{k}\left(v_{k}(x)\right) \leq M_{k} \quad \forall x \in \Omega .
$$

Vol. 37, $\left.\mathrm{N}^{\circ} 1,2019\right]$ 
Then, reasoning as in (24), we obtain

$$
\int_{\Omega}\left|g_{k}\left(v_{k}\right)\right|^{q} d x \leq C M_{k}^{q-1-\frac{1}{2^{\star}-1}} .
$$

From elliptic regularity (see (25)), we deduce

$$
\left\|v_{k}\right\|_{W^{2, q}(\Omega)} \leq C M_{k}^{1-\frac{1}{q}-\frac{1}{\left(2^{\star}-1\right) q}} .
$$

Therefore, from Morrey's Theorem (see (26)), for any $x \in B_{k}$ ),

$$
\left|v_{k}(x)-v_{k}\left(x_{k}\right)\right| \leq C\left(R_{k}\right)^{2-\frac{N}{q}} M_{k}^{1-\frac{1}{q}-\frac{1}{\left(2^{\star}-1\right) q}} .
$$

Particularizing $x=y_{k}$ in the above inequality, and from (27), we obtain

$$
C\left(R_{k}\right)^{2-\frac{N}{q}} M_{k}^{1-\frac{1}{q}-\frac{1}{\left(2^{\star}-1\right) q}} \geq\left|v_{k}\left(y_{k}\right)-v_{k}\left(x_{k}\right)\right|=\frac{1}{2}\left\|v_{k}\right\|,
$$

which implies

$$
\left(R_{k}\right)^{2-\frac{N}{q}} \geq \frac{1}{2 C} \frac{\left\|v_{k}\right\|}{M_{k}^{1-\frac{1}{q}-\frac{1}{\left(2^{\star}-1\right) q}}},
$$

or equivalently,

$$
R_{k} \geq\left(\frac{1}{2 C} \frac{\left\|v_{k}\right\|}{M_{k}^{1-\frac{1}{q}-\frac{1}{\left(2^{\star}-1\right) q}}}\right)^{1 /\left(2-\frac{N}{q}\right)}
$$

Consequently, taking into account (28),

$$
\int_{B_{k}} v_{k}\left|g_{k}\left(v_{k}\right)\right| d x \geq \frac{1}{2}\left\|v_{k}\right\| m_{k} \omega\left(R_{k}\right)^{N}
$$

where $\omega=\omega_{N}$ is the volume of the unit ball in $\mathbb{R}^{N}$.

Due to $B_{k} \subset \Omega$, substituting inequality (29), and rearranging terms, we obtain

$$
\begin{aligned}
& \int_{\Omega} v_{k}\left|g_{k}\left(v_{k}\right)\right| d x \geq \frac{1}{2}\left\|v_{k}\right\| m_{k} \omega\left(\frac{1}{2 C} \frac{\left\|v_{k}\right\|}{M_{k}^{1-\frac{1}{q}-\frac{1}{\left(2^{\star}-1\right) q}}}\right)^{\frac{N}{2-\frac{N}{q}}} \\
& =C m_{k}\left(\left[\left\|v_{k}\right\|\right]^{\frac{2}{N}-\frac{1}{q}} \frac{\left\|v_{k}\right\|}{M_{k}^{1-\frac{1}{q}-\frac{1}{\left(2^{\star}-1\right) q}}}\right)^{\frac{1}{\frac{2}{N}-\frac{1}{q}}} \\
& =C m_{k}\left(\frac{\left\|v_{k}\right\|^{1+\frac{2}{N}-\frac{1}{q}}}{M_{k}^{1-\frac{1}{q}-\frac{1}{\left(2^{\star}-1\right) q}}}\right)^{\frac{1}{N}-\frac{1}{q}} \\
& =C \frac{m_{k}}{M_{k}}\left(\frac{\left\|v_{k}\right\|^{1+\frac{2}{N}-\frac{1}{q}}}{M_{k}^{1-\frac{2}{N}-\frac{1}{\left(2^{\star}-1\right) q}}}\right)^{\frac{1}{\frac{2}{N}-\frac{1}{q}}} .
\end{aligned}
$$

[Revista Integración, temas de matemáticas 
At this moment, let us observe that from hypothesis $(\mathrm{H} 1)_{k}$ and $(\mathrm{H} 2)_{k}$,

$$
\frac{m_{k}}{M_{k}} \geq C, \quad \text { for all } \quad k \quad \text { big enough. }
$$

Hence, taking again into account hypothesis $(\mathrm{H} 2)_{k}$, and rearranging exponents, we can assert that

$$
\begin{aligned}
\int_{\Omega} v_{k}\left|g_{k}\left(v_{k}\right)\right| d x & \geq C\left(\frac{\left\|v_{k}\right\|^{1+\frac{2}{N}-\frac{1}{q}}}{M_{k}^{1-\frac{2}{N}-\frac{1}{\left(2^{\star}-1\right) q}}}\right)^{\frac{1}{\frac{2}{N}-\frac{1}{q}}} \\
& \geq C\left(\frac{\left\|v_{k}\right\|^{1+\frac{2}{N}-\frac{1}{q}}}{\left[g_{k}\left(\left\|v_{k}\right\|\right)\right]^{1-\frac{2}{N}-\frac{1}{\left(2^{\star}-1\right) q}}}\right)^{\frac{1}{\frac{2}{N}-\frac{1}{q}}} \\
& =C\left(\frac{\left\|v_{k}\right\|^{(N+2)\left[\frac{1}{N}-\frac{1}{(N+2) q}\right]}}{\left[g_{k}\left(\left\|v_{k}\right\|\right)\right]^{(N-2)\left[\frac{1}{N}-\frac{1}{(N+2) q}\right]}}\right)^{\frac{1}{\frac{2}{N}-\frac{1}{q}}} .
\end{aligned}
$$

Finally, from hypothesis $(\mathrm{F})_{k}$ we deduce

$$
\int_{\Omega} v_{k}\left|g_{k}\left(v_{k}\right)\right| d x \geq C\left(\frac{\left\|v_{k}\right\|^{2^{*}-1}}{g_{k}\left(\left\|v_{k}\right\|\right)}\right)^{\frac{(N-2)\left[\frac{1}{N}-\frac{1}{(N+2) q}\right]}{\frac{2}{N}-\frac{1}{q}}} \rightarrow \infty, \quad \text { as } \quad k \rightarrow \infty,
$$

which contradicts (23).

\subsection{Radial problems with almost critical exponent}

In this Section, we build an example of a sequence of functions $\left\{g_{k}\right\}$ growing subcritically, and satisfying the hypotheses $(\mathrm{H} 1)_{k},(\mathrm{H} 2)_{k}$, and $(\mathrm{F})_{k}$, such that the corresponding sequence of BVP

$$
\left\{\begin{aligned}
\Delta w_{k}+g_{k}\left(w_{k}\right)=0 & \text { in } \quad|x| \leq 1 \\
w_{k}(x)=0 & \text { for } \quad|x|=1 .
\end{aligned}\right.
$$

has an unbounded (in the $L^{\infty}(\Omega)$-norm) sequence $\left\{w_{k}\right\}$ of positive solutions. As a consequence of Theorem 4.2, this sequence $\left\{w_{k}\right\}$ is also unbounded in the $L^{2^{*}}(\Omega)$-norm.

Let $N \geq 3$ be an integer. For each positive integer $k>2$ let $g_{k}(s)=0$ for $s<0$; $g_{k}(s)=s^{\frac{N+2}{N-2}}$ for $s \in[0, k] ; g_{k}(s)=k^{\frac{N+2}{N-2}}$ for $s \in\left[k, k^{\frac{N+2}{N-2}}\right]$, and

$$
g_{k}(s)=k^{\frac{N+2}{N-2}}+\left(s-k^{\frac{N+2}{N-2}}\right)^{\frac{N+1}{N-2}}, \quad \forall s>k^{\frac{N+2}{N-2}} .
$$

Vol. 37, $\mathrm{N}^{\circ} 1,2019$ ] 
For the sake of simplicity in notation, we write $g_{k}:=g$.

Let $u_{k}:=u$ denote the solution to

$$
\left\{\begin{aligned}
u^{\prime \prime}+\frac{N-1}{r} u^{\prime}+g(u) & =0, & & r \in(0,1], \\
u(0) & =k^{\frac{N}{N-2}}, & & u^{\prime}(0)=0 .
\end{aligned}\right.
$$

Let $r_{1}=\sup \left\{r>0 ; u_{k}(s) \geq k\right.$ on $\left.[0, r]\right\}$. Since $g \geq 0, u$ is decreasing, consequently for $r \in\left[0, r_{1}\right], k \leq u(r) \leq k^{\frac{N}{N-2}}$, and

$$
-r^{N-1} u^{\prime}(r)=\int_{0}^{r} s^{N-1} g(u(s)) d s=\int_{0}^{r} s^{N-1} k^{\frac{N+2}{N-2}} d s=\frac{k^{\frac{N+2}{N-2}}}{N} r^{N},
$$

so

$$
-u^{\prime}(r)=\frac{k^{\frac{N+2}{N-2}}}{N} r
$$

Hence,

$$
u(r)=k^{\frac{N}{N-2}}-\frac{k^{\frac{N+2}{N-2}}}{2 N} r^{2}, \text { for } \quad r \in\left[0, r_{1}\right] .
$$

Thus, $u(r) \geq k^{\frac{N}{N-2}} / 2$, for all $0 \leq r \leq r_{0}:=\sqrt{N} / k^{\frac{1}{N-2}}$, and $u\left(r_{0}\right)=k^{\frac{N}{N-2}} / 2$.

By well established arguments based on the Pohozaev identity (see [18]), we have

$$
P(r):=r^{N} E(r)+\frac{N-2}{2} r^{N-1} u(r) u^{\prime}(r)=\int_{0}^{r} s^{N-1} \Gamma(u(s)) d s,
$$

where $E(r)=\frac{1}{2}\left(u^{\prime}(r)\right)^{2}+G(u(r)), \Gamma(s)=N G(s)-\frac{N-2}{2} s g(s)$, and $G(s)=\int_{0}^{s} g(t) d t$. For $s \in\left[k, k^{\frac{N}{N-2}}\right]$,

$$
\Gamma(s)=\frac{N+2}{2} k^{\frac{N+2}{N-2}}(s-k) \geq 0 .
$$

Hence,

$$
\Gamma(u(r)) \geq \frac{N+2}{2} k^{\frac{2 N+2}{N-2}} \text { for all } r \leq r_{0}, \quad k \geq 4^{\frac{N-2}{2}} .
$$

Due to $\Gamma(s)=0$ for all $s \leq k,(33)$ and (34), for $r \geq r_{0}$,

$$
P(r) \geq P\left(r_{0}\right) \geq \frac{N+2}{2 N} k^{\frac{2 N+2}{N-2}} r_{0}^{N}=\frac{N+2}{2} N^{\frac{N-2}{2}} k^{\frac{N+2}{N-2}} .
$$

From definition of $r_{1}$ and (32),

$$
\begin{aligned}
u\left(r_{1}\right) & =k, \text { with } \\
r_{1} & =\sqrt{2 N\left[\left(\frac{1}{k}\right)^{\frac{2}{N-2}}-\left(\frac{1}{k}\right)^{\frac{4}{N-2}}\right]} \\
& =\sqrt{2 N}\left(\frac{1}{k}\right)^{\frac{1}{N-2}}+o\left(\left(\frac{1}{k}\right)^{\frac{1}{N-2}}\right) .
\end{aligned}
$$


From definition of $g,-u^{\prime}\left(r_{1}\right)=\frac{1}{N} k^{\frac{N+2}{N-2}} r_{1}$ (see (31)), which implies

$$
\begin{aligned}
P\left(r_{1}\right) & \geq r_{1}^{N+2} O\left(k^{\frac{2(N+2)}{N-2}}\right)-r_{1}^{N} O\left(k^{\frac{2 N}{N-2}}\right) \\
& \geq O\left(k^{\frac{N+2}{N-2}}\right)-O\left(k^{\frac{N}{N-2}}\right) \\
& \geq O\left(k^{\frac{N+2}{N-2}}\right) .
\end{aligned}
$$

For $r \geq r_{1}$,

$$
\begin{aligned}
-\frac{N-2}{2} r^{N-1} u(r) u^{\prime}(r) & \geq \frac{(N-2) r^{N}}{2 N} u(r) u(r)^{\frac{N+2}{N-2}} \\
& =\frac{(N-2) r^{N}}{2 N} u(r)^{\frac{2 N}{N-2}} \\
& =r^{N} G(u(r)) .
\end{aligned}
$$

This, Pohozaev's identity, and (36) imply

$$
\left[\left(u^{\prime}(r)\right]^{2} \geq O\left(k^{\frac{N+2}{N-2}}\right) \frac{1}{r^{N}}, \quad \text { or } \quad-u^{\prime}(r) \geq O\left(k^{\frac{N+2}{2(N-2)}}\right) \frac{1}{r^{N / 2}} .\right.
$$

Integrating on $\left[r_{1}, r\right]$ we have

$$
u(r) \leq k-O\left(k^{\frac{N+2}{2(N-2)}}\right)\left(\frac{1}{r_{1}^{(N-2) / 2}}-\frac{1}{r^{(N-2) / 2}}\right),
$$

which implies that there exists $k_{0}$ such that if $k \geq k_{0}$ then $u(r)=0$ for some $r \in\left(r_{1}, 2 r_{1}\right]$. Since (37), $r_{1}=r_{1}(k) \rightarrow 0$ as $k \rightarrow \infty$.

Let $v:=v_{k}$ denote the solution to

$$
\left\{\begin{aligned}
v^{\prime \prime}+\frac{N-1}{r} v^{\prime}+g(v) & =0, & & r \in(0,1], \\
v(0) & =k^{\frac{N+2}{N-2}}, & & v^{\prime}(0)=0 .
\end{aligned}\right.
$$

Let $r_{1}^{\prime}=\sup \left\{r>0 ; v_{k}(s) \geq k\right.$ on $\left.[0, r]\right\}$. For $v(r) \geq k$, integrating (31) we deduce

$$
v(r)=k^{\frac{N+2}{N-2}}-\frac{k^{\frac{N+2}{N-2}}}{2 N} r^{2}, \text { for } \quad r \in\left[0, r_{1}^{\prime}\right]
$$

and

$$
v\left(r_{1}^{\prime}\right)=k^{\frac{N+2}{N-2}}-\frac{k^{\frac{N+2}{N-2}}}{2 N} r_{1}^{2}=k
$$

therefore,

$$
r_{1}^{\prime}=\sqrt{2 N\left(1-\left(\frac{1}{k}\right)^{\frac{4}{N-2}}\right)}>1,
$$

so $v(r) \geq k$ for all $r \in[0,1]$.

Vol. 37, $\left.\mathrm{N}^{\circ} 1,2019\right]$ 
Therefore, by continuous dependence on initial conditions, there exists $d_{k} \in$ $\left(k^{\frac{N}{N-2}}, k^{\frac{N+2}{N-2}}\right)$ such that the solution $w=w_{k}$ to

$$
\left\{\begin{aligned}
w^{\prime \prime}+\frac{N-1}{r} w^{\prime}+g_{k}(w) & =0, & & r \in(0,1], \\
w(0) & =d_{k}, & & w^{\prime}(0)=0,
\end{aligned}\right.
$$

satisfies $w(r) \geq 0$ for all $r \in[0,1]$, and $w(1)=0$. Since $k$ may be taken arbitrarily large, and as a consequence of Theorem 4.2, we have established the following result.

Corollary 4.4. There exists a sequence of functions $g_{k}: \mathbb{R} \rightarrow \mathbb{R}$ and a sequence $\left\{w_{k}\right\}$ of positive solutions to (30), such that each function $g_{k}$ grows subcritically and satisfies the hypotheses $(H 1)_{k},(H 2)_{k}$ and $(F)_{k}$ of Theorem 4.2, and the sequence $\left\{w_{k}\right\}$ of positive solutions to $(30)$ is unbounded in the $L^{\infty}(\Omega)$-norm.

Moreover, this sequence $\left\{w_{k}\right\}$ is also unbounded in the $L^{2^{*}}(\Omega)$-norm.

Let now $v:=v_{k}$ denote the solution to

$$
\left\{\begin{aligned}
v^{\prime \prime}+\frac{N-1}{r} v^{\prime}+g(v) & =0, & & r \in(0,1], \\
v(0) & =k, & & v^{\prime}(0)=0 .
\end{aligned}\right.
$$

Since $\Gamma(s)=0$ for all $s \leq k$, and the solution is decreasing, by Pohozaev's identity,

$$
\frac{1}{2} r\left(v^{\prime}(r)\right)^{2}+\frac{N-2}{2 N} r v(r)^{\frac{2 N}{N-2}}+\frac{N-2}{2} v(r) v^{\prime}(r)=0, \quad \forall r \in[0,1] .
$$

Hence, if $v(\hat{r})=0$ for some $\hat{r} \in(0,1]$, then $v^{\prime}(\hat{r})=0$ and the uniqueness of the solution of the IVP (38), implies $v(r)=0$ for all $r \in[0,1]$. Since this contradicts $v(0)=k>0$ we conclude that $v(r)>0$ for all $r \in[0,1]$. Therefore, by continuous dependence on initial conditions, there exists $d_{k}^{\prime} \in\left(k, k^{\frac{N}{N-2}}\right)$ such that the solution $z=z_{k}$ to

$$
\left\{\begin{aligned}
z^{\prime \prime}+\frac{N-1}{r} z^{\prime}+g_{k}(z) & =0, & & r \in(0,1], \\
z(0) & =d_{k}^{\prime}, & & z^{\prime}(0)=0,
\end{aligned}\right.
$$

satisfies $z(r) \geq 0$ for all $r \in[0,1]$, and $z(1)=0$.

Corollary 4.5. For any $k \in \mathcal{N}$, the BVP (30) has at least two positive solutions.

\section{Asymptotics for positive radial solutions of elliptic equations ap- proaching critical growth}

Consider the classical Dirichlet boundary value problem

$$
\begin{cases}-\Delta u=f(u) & \text { in } B_{R} \\ u>0 & \text { in } B_{R} \\ u=0 & \text { in } \partial B_{R}\end{cases}
$$


for $u \in C^{2}\left(\overline{B_{R}}\right)$, in which $B_{R}=B_{R}(0) \subset \mathbb{R}^{N}, N>2$, is the open ball of radius $R$, and $f$ is locally-Lipschitz in $[0, \infty)$ and superlinear at infinity (i.e. $\lim \inf f(u) / u>\lambda_{1}$ as $u \rightarrow \infty$, where $\lambda_{1}>0$ is the first eigenvalue of $-\Delta$ with Dirichlet boundary conditions). Denote by $2^{*}:=2 N /(N-2)$ the critical Sobolev exponent; $H^{1}(\Omega)$ is compactly embedded in $L^{p}(\Omega)$ if, and only if, $p<2^{*}$. The extended real number $f^{\star}:=\lim _{u \rightarrow \infty} f(u) / u^{2^{*}-1}$ discriminates the problem (39) into three types: critical if $f^{\star} \in(0, \infty)$, supercritical if $f^{*}=\infty$ and subcritical if $f^{\star}=0$.

Assume the nonlinearity is a pure subcritical power $f(u)=u^{2^{*}-1-\varepsilon}, \varepsilon>0$, and $\Omega$ is a ball. Atkinson and Peletier in [8] studied the assymptotic behavior as $\varepsilon \rightarrow 0^{+}$of solutions to $(39)$, and proved that

$$
\lim _{\varepsilon \rightarrow 0^{+}} \varepsilon u_{\varepsilon}(0)^{2}=L(N, R),
$$

and $\forall r \neq 0$,

$$
\lim _{\varepsilon \rightarrow 0^{+}} \frac{u_{\varepsilon}(r)}{\sqrt{\varepsilon}}=\widetilde{L}(N, R)\left(\frac{1}{r^{N-2}}-\frac{1}{R^{N-2}}\right),
$$

where $L(N, R), \widetilde{L}(N, R)$ are constants only dependent on $N$, and $R$, defined by

$$
\begin{aligned}
& L(N, R):=\frac{4}{N-2}[N(N-2)]^{\frac{N-2}{2}} \frac{\Gamma(N)}{\Gamma(N / 2)^{2}} \frac{1}{R^{N-2}} \\
& \widetilde{L}(N, R):=\frac{(N-2)^{\frac{1}{2}}}{2}[N(N-2)]^{\frac{N-2}{4}} \frac{\Gamma(N / 2)}{\Gamma(N)^{1 / 2}} R^{\frac{N-2}{2}}=\frac{[N(N-2)]^{\frac{N-2}{2}}}{L(N, R)^{1 / 2}}
\end{aligned}
$$

where $\Gamma$ denotes the Gamma function. See also [56] with similar results for least energy solutions on general domains.

The above sections lead to a natural question: Is the lower bound on $\alpha>\frac{2}{N-2}$ a technical or an intrinsic condition?

In the present Section, we focus our attention on nonlinearities

$$
f(u)=f_{\alpha}(u):=\frac{|u|^{2^{*}-2} u}{[\log (e+|u|)]^{\alpha}}, \quad \text { with } \alpha \in\left(0, \frac{2}{N-2}\right) .
$$

We analyze the asymptotic behavior as $\alpha \rightarrow 0^{+}$of solutions to (39). Firstly, we prove that for each $\alpha \in\left(0, \frac{2}{N-2}\right)$ fixed, the set of positive solutions to (39) is a priori bounded. Henceforth, the bound from below on $\alpha$ in [16], [17], [31], [71] are technical rather than intrinsic, at least when $\Omega$ is the open ball of radius $R$. Secondly, we provide estimates for the growth of $u_{\alpha}(0)$ as $\alpha \rightarrow 0^{+}$. We adapt for our nonlinearities, techniques introduced by Atkinson and Peletier for the case of subcritical powers in [7], [8].

Our first result is on the existence of solutions to (39), and of uniform $L^{\infty}$ a priori bounds for each $\alpha$ fixed (see [75, Proof of Theorem 1.1] for a proof).

Theorem 5.1. Fix $\alpha \in\left(0, \frac{2}{N-2}\right)$; let $f=f_{\alpha}$ be as in (42) and assume $\Omega$ is the open ball of radius $R$. Then the following results hold:

Vol. 37, $\left.\mathrm{N}^{\circ} 1,2019\right]$ 
(i) There exists a radially symmetric solution to (39), $u=u_{\alpha}(r)>0$.

(ii) There are constants $A=A_{\alpha}(N, R), B=B_{\alpha}(N, R)>0$ depending only on $\alpha, N$ and $R$, such that for every $u=u_{\alpha}>0$, radially symmetric solution to (39), we have

$$
A_{\alpha}(N, R) \leq\left\|u_{\alpha}\right\|_{L^{\infty}(\Omega)} \leq B_{\alpha}(N, R), \quad \text { for each } \alpha \in\left(0, \frac{2}{N-2}\right) .
$$

Our second result is an estimate of the asymptotic behavior of $u_{\alpha}(0)=\left\|u_{\alpha}\right\|_{L^{\infty}(\Omega)}$ as $\alpha \rightarrow 0^{+}$.

Theorem 5.2. Let $f=f_{\alpha}$ be as in (42) with $\alpha \in\left(0, \frac{2}{N-2}\right)$, and $\Omega$ be the open ball of radius $R$. Then, there exists a constant $L(N, R)>0$ only depending on $N$ and $R$, such that for any $u_{\alpha}=u_{\alpha}(r)$, radially symmetric positive solution to (39), we have

$$
\begin{aligned}
\limsup _{\alpha \rightarrow 0^{+}} \frac{\alpha u_{\alpha}(0)^{2}}{\left[\log \left(e+u_{\alpha}(0)\right)\right]^{1+\frac{\alpha(N+2)}{2}}} \leq L(N, R), \\
\liminf _{\alpha \rightarrow 0^{+}} \frac{\alpha u_{\alpha}(0)^{2}}{\left[\log \left(e+u_{\alpha}(0)\right)\right]^{\frac{\alpha(N-4)}{2}}} \geq \frac{1}{2^{*}} L(N, R),
\end{aligned}
$$

where $L(N, R)$ is defined by $(40)$.

Proof. See [75, Proof of Theorem 1.2].

\section{Some Open Problems}

- When $N=2$, which is the critical nonlinearity $f$ for having a priori bounds? (See [38], [39]).

- When $N>2$, which is the more general subcritical nonlinearity $f$ for having $a$ priori bounds?

- When $p=N$, which is the critical nonlinearity $f$ for having a priori bounds? (See [36]).

- When $N>p$, which is the more general subcritical nonlinearity $f$ for having $a$ priori bounds?

(See [36]).

- The conjecture on Lane-Emdem systems when $\Omega=\mathbb{R}^{N}$.

See the discussion corresponding to the Lane-Emdem system (7).

\section{Acknowledgements}

The author was partially supported by Grant MTM2016-75465, MINECO, Spain and Grupo de Investigación CADEDIF 920894, UCM.

[Revista Integración, temas de matemáticas 


\section{References}

[1] Agmon S., Douglis A. and Nirenberg L.,"Estimates near the boundary for solutions of elliptic partial differential equations satisfying general boundary conditions, I", Comm. Pure Appl. Math. 12 (1959), 623-727.

[2] Agmon S., Douglis A. and Nirenberg L., "Estimates near the boundary for solutions of elliptic partial differential equations satisfying general boundary conditions, II", Comm. Pure Appl. Math. 17 (1964), 35-92.

[3] Alexander J.C. and Antman S.S., "Global and local behavior of bifurcating multidimensional continua of solutions for multiparameter nonlinear eigenvalue problems", Arch. Rational Mech. Anal. 76 (1981), No. 4, 339-354.

[4] Allegretto W. and Huang Y.X., "A Picone's identity for the p-Laplacian and applications", Nonlinear Anal. 32 (1998), No. 7, 819-830.

[5] Anane A., "Simplicité et isolation de la première valeur propre du p-laplacien avec poids", C.R. Acad. Sci. Paris. Sér. I Math. 305 (1987), No. 16, 725-728.

[6] Astarita G. and Marrucci G., Principles of Non-Newtonian Fluid Mechanics, McGraw Hill, New York, 1974.

[7] Atkinson F.V. and Peletier L.A., "Emden-Fowler equations involving critical exponents", Nonlinear Anal. 10 (1986), No. 8, 755-776.

[8] Atkinson F.V. and Peletier L.A., "Elliptic equations with nearly critical growth", J. Differential Equations 70 (1987), No. 3, 349-365.

[9] Azizieh C. and Clément P., "A priori estimates and continuation methods for positive solutions of p-Laplace equations", J. Differential Equations 179 (2002), No. 1, $213-245$.

[10] Azizieh C., Clément P. and Mitidieri E., "Existence and a priori estimates for positive solutions of p -Laplace systems", J. Differential Equations 184 (2002), No. $2,422-442$.

[11] Birindelli I. and Mitidieri E., "Liouville theorems for elliptic inequalities and applications", Proc. Roy. Soc. Edinburgh Sect. A 128 (1998), No. 6, 1217-1247.

[12] Bonheure D., dos Santos E.M. and Tavares H., "Hamiltonian elliptic systems: a guide to variational frameworks", Port. Math. 71 (2014), No. 3-4, 301-395.

[13] Brezis H., Functional analysis, Sobolev spaces and partial differential equations, Universitext, Springer, New York, 2011.

[14] Busca J. and Manásevich R., "A Liouville-type Theorem for Lane-Emden Systems", Indiana Univ. Math. J. 51 (2002), No. 1, 37-51.

[15] Castro A., Mavinga N. and Pardo R., "Equivalence between uniform $L^{2^{\star}}(\Omega)$ a-priori bounds and uniform $L^{\infty}(\Omega)$ a-priori bounds for subcritical elliptic equations", To appear in Topol. Methods Nonlinear Anal. (2019).

Vol. 37, $\mathrm{N}^{\circ}$ 1, 2019] 
[16] Castro A. and Pardo R., "A priori bounds for positive solutions of subcritical elliptic equations", Rev. Mat. Complut. 28 (2015), No. 3, 715-731.

[17] Castro A. and Pardo R., "A priori estimates for positive solutions to subcritical elliptic problems in a class of non-convex regions", Discrete Contin. Dyn. Syst. Ser. B 22 (2017), No. 3, 783-790.

[18] Castro A. and Kurepa A., "Infinitely many radially symmetric solutions to a superlinear Dirichlet problem in a ball", Proc. Amer. Math. Soc. 101 (1987), No. 1, $57-64$.

[19] Cianchi A. and Maz'ya W., "Gradient regularity via rearrangements for $p$-Laplacian type elliptic boundary value problems", J. Eur. Math. Soc. 16 (2014), No.3, 571595.

[20] Cianchi A. and Maz'ya W., "Global gradient estimates in elliptic problems under minimal data and domain regularity", Commun. Pure Appl. An. 14 (2015), No.1, $285-311$.

[21] Clement Ph., de Figueiredo D.G. and Mitidieri E., "Positive Solutions of Semilinear Elliptic Systems", Comm. Partial Differential Equations 17 (1992), No. 5-6, 923940.

[22] Clement Ph., de Pagter B., Sweers G. and de Thelin F., "Existence of Solutions to a Semilinear Elliptic System through Orlicz-Sobolev Spaces", Mediterr. J. Math. 1 (2004), No. 3, 241-267.

[23] Cosner C., "Positive Solutions for Superlinear Elliptic Systems, without variational structure", Nonlinear Anal. 8 (1984), No. 12, 1427-1436.

[24] Crandall M.G. and Rabinowitz P.H., "Bifurcation from simple eigenvalues", $J$. Functional Analysis 8 (1971), 321-340.

[25] Cuesta M. and Takak P., "A strong comparison principle for the Dirichlet $p$ laplacian", in Lecture notes in Pure and Appl. Math. 194, Dekker, New York (1998), $79-87$.

[26] D'Ambrosio L. and Mitidieri E., "A priori estimates, positivity results, and nonexistence theorems for quasilinear degenerate elliptic inequalities", Adv. Math. 224 (2010), No. 3, 967-1020.

[27] D'Ambrosio L. and Mitidieri E., "Liouville theorems for elliptic systems and applications", J. Math. Anal. Appl. 413 (2014), No. 1, 121-138.

[28] Damascelli L., "Comparison theorems for some quasilinear degenerate elliptic operators and applications to symmetry and monotonicity results", Ann. Inst. H. Poincaré. Analyse non linéaire 15 (1998), No. 4, 493-516.

[29] Damascelli L. and Pacella F., "Monotonicity and symmetry of solutions of $p$-Laplace equations, $1<p<2$, via the moving plane method", Ann. Scuola Norm. Sup. Pisa Cl. Sci (4) 26 (1998), No. 4, 689-707.

[Revista Integración, temas de matemáticas 
[30] Damascelli L. and Pacella F., "Monotonicity and symmetry results for $p$-Laplace equations and applications", Adv. Differential Equations 5 (2000), No. 7-9, 11791200 .

[31] Damascelli L. and Pardo R., "A priori estimates for some elliptic equations involving the p-Laplacian", Nonlinear Anal. Real World Appl. 41 (2018), 475-496.

[32] Damascelli L. and Sciunzi B., "Regularity, monotonicity and symmetry of positive solutions of $m$-Laplace equations", J. Differential Equations 206 (2004), No. 2, $483-515$.

[33] Damascelli L. and Sciunzi B., "Harnack inequalities, maximum and comparison principles, and regularity of positive solutions of m-Laplace equations", Calc. Var. Partial Differential Equations 25 (2006), No. 2, 139-159.

[34] Dancer E.N., "On the structure of solutions of non-linear eigenvalue problems", Indiana Univ. Math. J. 23 (1973/74), 1069-1076.

[35] Dancer E.N., "Bifurcation from simple eigenvalues and eigenvalues of geometric multiplicity one", Bull. London Math. Soc. 34 (2002), No. 5, 533-538.

[36] De Figueiredo D.G., do Ó J.M. and Ruf B., "On an Inequality by N. Trudinger and J. Moser and Related Elliptic Equations", Comm. Pure Appl. Math. 55 (2002), No. $2,135-152$.

[37] De Figueiredo D.G., do Ó J.M. and Ruf B., "An Orlicz-space approach to superlinear elliptic systems", J. Funct. Anal. 224 (2005), No. 2, 471-496.

[38] De Figueiredo D.G., do Ó J.M. and Ruf B., "Semilinear Elliptic Systems With Exponential Nonlinearities in Two Dimensions", Adv. Nonlinear Stud. 6 (2006), No. 2, 199-213.

[39] De Figueiredo D.G., do Ó J.M. and Ruf B., "Non-variational elliptic systems in dimension two: a priori bounds and existence of positive solutions", J. Fixed Point Theory Appl. 4 (2008), No. 1, 77-96.

[40] De Figueiredo D.G., Lions P.-L. and Nussbaum R.D., "A priori estimates and existence of positive solutions of semilinear elliptic equations", J. Math. Pures Appl. (9) 61 (1982), No. 1, 41-63.

[41] DiBenedetto E., " $C^{1+\alpha}$ local regularity of weak solutions of degenerate elliptic equations", Nonlinear Anal. 7 (1983), No. 8, 827 -850.

[42] Farina A. and Serrin J., "Entire solutions of completely coercive quasilinear elliptic equations I", J. Differential Equations 250 (2011), No. 12, 4367-4408.

[43] Farina A. and Serrin J., "Entire solutions of completely coercive quasilinear elliptic equations II", J. Differential Equations 250 (2011), No. 12, 4409-4436.

[44] Farina A., Montoro L. and Sciunzi B., "Monotonicity and one-dimensional symmetry for solutions of $-\Delta_{p} u=f(u)$ in half-spaces", Calc. Var. Part. Diff. Eq. 43 (2012), No. 1, 123-145.

Vol. 37, $\left.\mathrm{N}^{\circ} 1,2019\right]$ 
[45] Farina A., Montoro L. and Sciunzi B., "Monotonicity of solutions of quasilinear degenerate elliptic equations in half-spaces", Math. Ann. 357 (2013), No. 3, 855893.

[46] Farina A., Montoro L. and Sciunzi B., "Monotonicity in half-spaces of positive solutions of $-\Delta_{p} u=f(u)$ in the case $p>2$ ", Ann. Sc. Norm. Super. Pisa Cl. Sci. (5) 17 (2017), No. 4, 1207-1229.

[47] Farina A., Montoro L., Riey G. and Sciunzi B., "Monotonicity of solutions to quasilinear problems with a first-order term in half-spaces", Ann. Inst. H. Poincaré Anal. Non Linéaire 32 (2015), No. 1, 1-22.

[48] Fleckinger J. and Pardo R., "Bifurcation for an elliptic system coupled in the linear part", Nonlinear Anal. RWA 37 (1999), No. 1, 13-30.

[49] Fleckinger J., Pardo R. and de Thélin F., "Four-parameter bifurcation for a $p$ Laplacian system", Electron. J. Differential Equations (2001), No. 6, 1-15.

[50] Garcia Azorero J. and Peral I., "Existence and nonuniqueness for the p-laplacian: Nonlinear eigenvalues", Comm. Partial Differential Equations 12 (1987), No. 12, 1389-1430.

[51] Gidas B., Ni W.M. and Nirenberg L., "Symmetry and related properties via the maximum principle", Comm. Math. Phys. 68 (1979), No. 3, 209-243.

[52] Gidas B. and Spruck J., "A priori bounds for positive solutions of nonlinear elliptic equations", Comm. Partial Differential Equations 6 (1981), No. 8, 883-901.

[53] Gidas B. and Spruck J., "Global and local behavior of positive solutions of nonlinear elliptic equations", Comm. Pure Appl. Math. 34 (1981), No. 4, 525-598.

[54] Gilbarg D. and Trudinger N.S., Elliptic partial differential equations of second order, volume 224 of Grundlehren der Mathematischen Wissenschaften [Fundamental Principles of Mathematical Sciences], Springer-Verlag, Berlin, 1983.

[55] Guedda M. and Veron L., "Quasilinear elliptic equations involving critical Sobolev exponents",Nonlinear Anal. 13 (1989), No. 8, 879-902.

[56] Han Z.-C., "Asymptotic approach to singular solutions for nonlinear elliptic equations involving critical Sobolev exponent", Ann. Inst. H. Poincaré Anal. Non Linéaire 8 (1991), No. 2, 159-174.

[57] Iwaniek T., "Projections onto gradient fields and $L^{p}$ estimates for degenerated elliptic operators", Studia Math. 75 (1983), No. 3, 293-312.

[58] Krasnoselskii M.A., "Fixed point of cone-compressing or cone-extending operators", Soviet Math. Dokl. 1 (1960), 1285-1288.

[59] Ladyzhenskaya O.A. and Ural'tseva N.N.,Linear and quasilinear elliptic equations, Academic Press, New York-London, 1968.

[60] Lieberman G.M., "Boundary regularity for solutions of degenerate elliptic equations", Nonlinear Anal. 12 (1988), No. 11, 1203-1219.

[Revista Integración, temas de matemáticas 
[61] Lindqvist P., "On the equation $\Delta_{p} u+\lambda|u|^{p-2} u=0$ ", Proc. A.M.S. 109 (1990), No. $1,157-164$.

[62] Lions P.-L., "On the existence of positive solutions of semilinear elliptic equations", SIAM Rev. 24 (1982), No. 4, 441-467.

[63] López-Gómez J., Spectral theory and nonlinear functional analysis, Chapman \& Hall/CRC, Boca Raton, FL, 2001.

[64] López-Gómez J. and Pardo R., "Multiparameter nonlinear eigenvalue problems: positive solutions to elliptic Lotka-Volterra systems", Appl. Anal. 31 (1988), No. $1-2,103-127$.

[65] López-Gómez J. and Pardo R., "Existence and uniqueness for some competition models with diffusion", C.R. Acad. Sci. Paris Sér. I Math. 313 (1991), No. 13, 933-938.

[66] López-Gómez J. and Pardo R., "Coexistence regions in Lotka-Volterra models with diffusion", Nonlinear Anal. 19 (1992), No. 1, 11-28.

[67] López-Gómez J. and Pardo R., "Coexistence in a simple food chain with diffusion", J. Math. Biol. 30 (1992), No. 7, 655-668.

[68] López-Gómez J. and Pardo R., "Invertibility of linear noncooperative elliptic systems", Nonlinear Anal. 31 (1998), No. 5-6, 687-699.

[69] Marinson L.K. and Pavlov K.B., "The effect of magnetic plasticity in nonNewtonian fluids", Magnit. Gidrodinamika 3 (1969), 69-75.

[70] Marinson L.K. and Pavlov K.B., "Unsteady shear flows of a conducting fluid with a rheological power law", Magnit. Gidrodinamika 2 (1970), 50-58.

[71] Mavinga N. and Pardo R., "A priori bounds and existence of positive solutions for subcritical semilinear elliptic systems", J. Math. Anal. Appl. 449 (2017), No. 2, $1172-1188$.

[72] Mitidieri E., "A Rellich type identity and applications", Comm. Partial Differential Equations 18 (1993), No.1-2, 125-151.

[73] Mitidieri E. and Pohozaev S.I., "Absence of global positive solutions of quasilinear elliptic inequalities", Dokl. Akad. Nauk. 359 (1998), No. 4, 456-460. [In Russian].

[74] Mitidieri E. and Pohozaev S.I., "Nonexistence of positive solutions for quasilinear

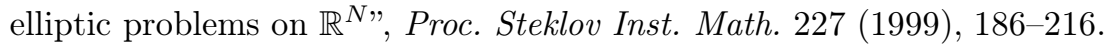

[75] Pardo R. and Sanjuán A., "Asymptotics for positive radial solutions of elliptic equations approaching critical growth", Preprint.

[76] Peletier L.A. and Van der Vorst R.C.A.M., "Existence and nonexistence of positive solutions of nonlinear elliptic systems and the biharmonic equation", Differential Integral Equations 5 (1992), No. 4, 747-767.

[77] Peral I., Multiplicity of solutions for the p-Laplacian, ICTP lectures, Madrid, 1997.

Vol. 37, $\left.\mathrm{N}^{\circ} 1,2019\right]$ 
[78] Pucci P. and Serrin J., The maximum principle, Birkhäuser Verlag, Basel, 2007.

[79] Quittner P. and Souplet P.H., "A priori estimates and existence for elliptic systems via bootstrap in weighted Lebesgue spaces", Arch. Ration. Mech. Anal. 174 (2004), No. 1, 49-81.

[80] Quittner P., "A priori estimates, existence and Liouville theorems for semilinear elliptic systems with power nonlinearities", Nonlinear Anal. 102 (2014), 144-158.

[81] Rabinowitz P.H., "Some aspects of nonlinear eigenvalue problems", Rocky Mountain J. Mat. 3 (1973), 161-202.

[82] Rabinowitz P.H., "Some global results for nonlinear eigenvalue problems", J. Funct. Anal. 7 (1971), 487-513.

[83] Ruiz D., " A priori estimates and existence of positive solutions for strongly nonlinear problems", J. Differential Equations 199 (2004), No. 1, 96-114.

[84] Serrin J. and Zou H., "Cauchy-Liouville and universal boundedness theorems for quasilinear elliptic equations and inequalities", Acta Math. 189 (2002), No. 1, 79142.

[85] Serrin J. and Zou H., "Existence of positive solutions of Lane-Emden systems", Atti Sem. Mat. Fis. Univ. Modena 46 (1998), 369-380.

[86] Serrin J. and Zou H., "Existence of entire positive solutions of elliptic Hamiltonian systems", Comm. Partial Differential Equations 23 (1998), No. 3-4, 577-599.

[87] Souplet P.H., "The proof of the Lane-Emden conjecture in four space dimensions", Adv. Math. 221 (2009), No. 5, 1409-1427.

[88] Tolksdorf P., "Regularity for a more general class of quasilinear elliptic equations", J. Differential Equations 51 (1984), No. 1, 126-150.

[89] Troy W.C., "Symmetry properties in systems of semilinear elliptic equations", J. Differential Equations 42 (1981), No. 3, 400-413.

[90] Trudinger N., "Remarks concerning the conformal deformation of Riemannian structures on compact manifolds", Ann. Scuola Norm. Sup. Pisa (3) 22 (1968), $265-274$.

[91] Vazquez J.L., "A strong maximum principle for some quasilinear elliptic equations", Appl. Math. Optim. 12 (1984), No. 3, 191-202.

[92] Zou H.H., "A priori estimates and existence for quasi-linear elliptic equations", Calc. Var. Partial Differential Equations 33 (2008), No. 4, 417-437.

[93] Zou H., "A priori estimates for a semilinear elliptic system without variational structure and their applications", Math. Ann. 323 (2002), No. 4, 713-735. 